\title{
A Mathematical Model for Flood Protection
}

\author{
C. $\operatorname{Roos}^{1}$
}

\begin{abstract}
Many regions in the world are protected against flooding by a dike, which may be either natural or artificial. We deal with a model for finding the optimal heights of such a dike in the future. It minimizes the sum of the investments costs for upgrading the dike in the future and the expected costs due to flooding. The model is highly nonlinear, nonconvex, and infinite-dimensional. Despite this, the model can be solved analytically if there is no backlog in maintenance. If there is a backlog in maintenance, then the optimal solution can be found by minimizing a convex function over a finite interval. However, if the backlog becomes extremely large we show that the model breaks down. Our model has been used in The Netherlands to define legal safety standards for the coming decades.
\end{abstract}

Keywords: Flood prevention, Cost-benefit analysis, Infinite dimensional optimization.

Manuscript was received on 12/09/2017, revised on 03/12/2017 and accepted for publication on 05/12/2017.

\section{Introduction}

Dike height optimization is of major importance to the Netherlands because a large part of the country lies below sea level and high water levels in rivers can also cause floods. After a devastating flood in the Netherlands in 1953, Van Dantzig proposed a cost-benefit model to protect The Netherlands against new floods [6]. His model is considered to be the first real-life application of Operations Research techniques in The Netherlands.

In 2006, Van Dantzig's model was improved by Eijgenraam [4]. He showed numerically the shortcomings of Van Dantzig's model, which contrary to the new model did not take into account the growth of economy in the protected area during time.

The submersible part of The Netherlands is divided in more than 50 so-called dike rings. A dike ring is an area that is surrounded by a dike. Most dike rings consist of several segments that are characterized by different properties. Eijgenraam's model focused on a one-segment (or homogeneous) dike. He found a nice periodic solution of his model and claimed that this solution is optimal. The proof of this claim in [4] has a flaw, however.

Some attempts were made to generalize Eijgenraam's model to the case of nonhomogeneous dikes, i.e., dikes with more than one segment. In such a case, an analytic solutions seems to be out of reach. As made clear in [5], also some initial numerical attempts to deal with nonhomogeneous dikes failed.

In some recent papers, we dealt with Eijgenraam's model and its generalization to the nonhomogeneous case $[1,2,3]$. In [1, 3], we focussed on a nonhomogeneous extension of

\footnotetext{
${ }^{1}$ Department of Electrical Engineering, Computer Science and Mathematics, Delf University of Technology, Delf, The Netherlands, Email: c.roos@ tudelf.nl.
} 
Eijgenraam's model for which we developed a mixed integer nonlinear model that could be solved by CPLEX, while using CONOPT to deal with nonlinearity. In [2], we gave a full analysis of the homogeneous model of Eijgenraam. The model is a highly nonlinear, nonconvex, and infinitedimensional optimization problem. Despite this, we could show that the periodic solution of Eijgenraam's model is optimal, provided that there is no backlog in maintenance. We called a dike healthy in that case; otherwise, unhealthy. We also showed that if a dike is unhealthy, then it is optimal to upgrade the dike immediately with a specific quantity which makes the dike healthy; after this upgrade, the dike behaves as a healthy dike, and the optimal upgrade policy is then again periodic.

In this paper, we only deal with homogeneous dikes. We present a simplified and streamlined proof that if the dike is healthy, then the solution of Eijgenraam's model is periodic, as we showed earlier in the (electronic) appendix of [2]. The main focus, however, is on unhealthy dikes. We show that if the expected damage is too large, in fact extremely large, then the model breaks down, because then it becomes attractive to split an upgrade at some time in two separate upgrades at the same time. Since the set-up costs in our model are positive, this is absurd.

It may be clear that our model may also be applicable to other deltas in the world where the ground is sinking and/or the water level is rising.

\section{Mathematical Model for a Homogeneous Dike}

Our model uses the following functions and parameters:

$H_{t}: \quad$ dike height at time $t$,

$P_{t}=P_{0} e^{a \eta t} e^{-a\left(H_{t}-H_{0}\right)}$ : flood probability at time $t$,

$\alpha: \quad$ parameter in exponential distribution for extreme water levels $(1 / \mathrm{cm})$,

$\eta$ : $\quad$ ground level decrease plus water level increase ( $\mathrm{cm} /$ year),

$V_{t}=V_{0} e^{\gamma t} e^{\zeta\left(H_{t}-H_{0}\right)}$ : loss by flooding at time $t$ (million euros),

$\gamma: \quad$ rate of growth of wealth in dike ring (per year),

$\zeta: \quad$ increase of loss per $\mathrm{cm}$ dike heightening $(1 / \mathrm{cm})$,

$\delta: \quad$ discount rate (1/year).

The expected loss at time $t$ is then

$$
S_{t}=P_{t} V_{t}=P_{0} e^{\alpha \eta t} e^{-\alpha\left(H_{t}-H_{0}\right)} \cdot V_{0} e^{\gamma t} e^{\zeta\left(H_{t}-H_{0}\right)}=S_{0} e^{\beta_{1} t} e^{-\theta h_{t}},
$$

where

$$
S_{0}=P_{0} V_{0}, \quad \beta_{1}=\alpha \eta+\gamma, \quad \theta=\alpha-\zeta, \quad h_{t}=H_{t}-H_{0} .
$$

We assume that upgrades of the dike take place at moments $t_{k}, k=1,2, \ldots$. The value of the upgrade at moment $t_{k}$ is denoted as $u_{k}$. We require that

$$
u_{k}>0, \quad t_{k+1}>t_{k} \geq t_{0}=0, \quad k \geq 1
$$

The height at moment $t_{k}$ (including $u_{k}$ ) is denoted as $h_{k}$. We then have

$$
h_{0}=0, \quad h_{k}=h_{k-1}+u_{k}, \quad k \geq 1 \text {. }
$$




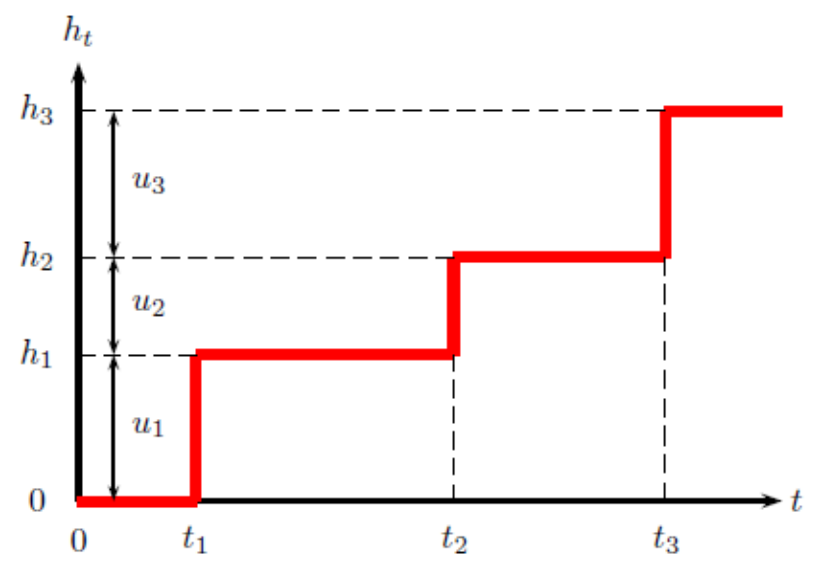

Figure 1. Graph of $h_{t}$

We define infinite sequences $u, h$ and $\tau$ as follows:

$$
\begin{aligned}
u & :=\left(u_{1} ; u_{2} ; u_{3} ; \ldots\right), \\
h & :=\left(h_{1} ; h_{2} ; h_{3} ; \ldots\right), \\
\tau & :=\left(t_{1} ; t_{2} ; t_{3} ; \ldots\right) .
\end{aligned}
$$

It is clear that if we know $u$, then $h$ follows from (2), and vice versa. So, we may consider the total costs as a function $f(u, \tau)$ of $u$ and $\tau$ alone. One has

$$
f(u, \tau)=I(u, \tau)+A(u, \tau)
$$

where $I(u, \tau)$ represents the total investment costs, and $A(u, \tau)$ is the total expected damage. The costs for an upgrade of the dike with $u_{k}$ at $t=t_{k}$ are given by

$$
D\left(u_{k}\right) e^{\lambda h_{k}} e^{-\delta t_{k}}, \quad D\left(u_{k}\right)=c+b u_{k}
$$

where the factor $e^{-\delta t_{k}}$ takes care of discounting future costs to present costs. We may now write

$$
\begin{aligned}
I(u, \tau) & =\sum_{k=1}^{\infty} D\left(u_{k}\right) e^{\lambda h_{k}-\delta t_{k}}, \\
A(u, \tau) & =\sum_{k=1}^{\infty} \int_{t_{k-1}}^{t_{k}} S_{t} e^{\delta t} d t=\sum_{k=1}^{\infty} \int_{t_{k-1}}^{t_{k}} S_{0} e^{\beta_{1} t} e^{-\theta\left(H_{t}-H_{0}\right)} e^{-\delta t} d t \\
& =\sum_{k=1}^{\infty} \frac{S_{0}}{\beta}\left[e^{\beta t_{k}}-e^{\beta t_{k-1}}\right] e^{-\theta h_{k-1}},
\end{aligned}
$$

where $\beta=\beta_{1}-\delta$ and where we used that the height of the dike in the interval $\left[t_{k-1}, t_{k}\right)$ equals $h_{k-1}$. We call the pair $(u, \tau)$ admissible if (1) and (2) are satisfied. Our aim is to find an admissible pair $(u, \tau)$ that minimizes $f(u, \tau)$.

It will be assumed that 


$$
S_{0}>0, \quad b \geq 0, \quad c>0, \quad \lambda \geq 0, \quad \delta>0, \quad \theta>0, \quad \beta \neq 0,
$$

thereby we may use that

$$
\beta+\delta>0, \quad \theta \delta-\lambda \beta>0, \quad \lambda+b>0,
$$

because these inequalities are satisfied for all dike rings in The Netherlands. The assumption $\beta \neq 0$ is only made to simplify the presentation; the arguments used below can easily be adapted to the case where $\beta=0$.

Note that if $K$ is any positive constant and we redefine $b, c$ and $S_{0}$ according to $b:=K b, c:=K c$ and $S_{0}:=K S_{0}$, then the values of $I(u, \tau)$ and $A(u, \tau)$ are multiplied by $K$. So, by taking $K=1 / c$, we may normalize the problem such that $c=1$.

\section{First Order Optimality Conditions with Respect to $t_{k}$}

By computing the partial derivative of $f(u, \tau)$ with respect to $t_{k}$, we obtain

$$
\frac{\partial f(u, \tau)}{\partial t_{k}}=-\delta D\left(u_{k}\right) e^{\lambda h_{k}-\delta t_{k}}+S_{0} e^{\beta t_{k}-\theta h_{k-1}}-S_{0} e^{\beta t_{k}-\theta h_{k}}
$$

Hence, the pair $(u, \tau)$ is stationary with respect to $t_{k}$ if and only if

$$
\delta D\left(u_{k}\right) e^{\lambda h_{k}-\delta t_{k}}=S_{0} e^{\beta t_{k}-\theta h_{k-1}}-S_{0} e^{\beta t_{k}-\theta h_{k}}, \quad k \geq 1 .
$$

Because of (2), this condition can be written as

$$
\delta D\left(u_{k}\right) e^{(\lambda+\theta) h_{k}-(\beta+\delta) t_{k}}=S_{0}\left(e^{\theta u_{k}}-1\right) .
$$

We define

$$
\varrho(x):=\ln \frac{\delta D(x)}{S_{0}\left(e^{\theta x}-1\right)}, \quad x>0 .
$$

By taking logarithms of both sides of (6) the condition for stationarity with respect to $t_{k}$ becomes

$$
\varrho\left(u_{k}\right)=(\beta+\delta) t_{k}-(\lambda+\theta) h_{k}, \quad k \geq 1
$$

As we already established, if $u$ is known, then $h$ can be computed. The above relation reveals that then also the sequence $\tau$ can be computed, because since $\beta+\delta>0$ we may write

$$
t_{k}=\frac{\varrho\left(u_{k}\right)+(\lambda+\theta) h_{k}}{\beta+\delta} .
$$

Hence, a stationary pair $(u, \tau)$ is completely determined by its sequence $u$. Therefore, we call the sequence $u$ a stationary sequence if the corresponding $(u, \tau)$ is stationary. Similarly, $u$ is said to be admissible (optimal) if $(u, \tau)$ is admissible (optimal). 
In the sequel, we also use the function

$$
\kappa(x):=\frac{D(x)}{e^{\theta x}-1}, \quad x>0
$$

It is related to the function $\varrho(x)$ via

$$
S_{0} e^{\varrho(x)}=\frac{\delta D(x)}{e^{\theta x}-1}=\delta \kappa(x) .
$$

This yields a second way to characterize stationarity with respect to $t_{k}$, namely,

$$
S_{0} e^{(\beta+\delta) t_{k}}=\delta \kappa\left(u_{k}\right) e^{(\theta+\lambda) h_{k}}
$$

\section{First Order Optimality Conditions with Respect to $\boldsymbol{u}_{k}$}

Since $h_{\ell}=\sum_{k=1}^{\ell} u_{k}$, we have

$$
\frac{\partial h_{\ell}}{\partial u_{k}}= \begin{cases}1, & \text { if } \ell \geq k \\ 0, & \text { if } \ell<k\end{cases}
$$

Using this we get, for $k \geq 1$,

$$
\frac{\partial f(u, \tau)}{\partial u_{k}}=b e^{\lambda h_{k}-\delta t_{k}}+\sum_{\ell \geq k} \lambda D\left(u_{\ell}\right) e^{\lambda h_{\ell}-\delta t_{\ell}}-\sum_{\ell-1 \geq k} \frac{\theta S_{0}}{\beta}\left(e^{\beta t_{\ell}}-e^{\beta t_{\ell-1}}\right) e^{-\theta h_{\ell-1}} .
$$

It follows that stationarity with respect to $u_{k}$ is equivalent to

$$
b e^{\lambda h_{k}-\delta t_{k}}+\sum_{\ell=k}^{\infty} \lambda D\left(u_{\ell}\right) e^{\lambda h_{\ell}-\delta t_{\ell}}=\sum_{\ell=k+1}^{\infty} \frac{\theta S_{0}}{\beta}\left(e^{\beta t_{\ell}}-e^{\beta t_{\ell-1}}\right) e^{\theta h_{\ell-1}}, \quad k \geq 1 .
$$

This, in turn, can also be written as

$$
b e^{\lambda h_{k+1}-\delta t_{k+1}}+\sum_{\ell=k+1}^{\infty} \lambda D\left(u_{\ell}\right) e^{\lambda h_{\ell}-\delta t_{\ell}}=\sum_{\ell=k+2}^{\infty} \frac{\theta S_{0}}{\beta}\left(e^{\beta t_{\ell}}-e^{\beta t_{\ell-1}}\right) e^{\theta h_{\ell-1}}, \quad k \geq 0 .
$$

Subtracting these equations from each other, we get

$$
b e^{\lambda h_{k}-\delta t_{k}}+\lambda D\left(u_{k}\right) e^{\lambda h_{\ell}-\delta t_{k}}-b e^{\lambda h_{k+1}-\delta t_{k+1}}=\frac{\theta S_{0}}{\beta}\left(e^{\beta t_{k+1}}-e^{\beta t_{k}}\right) e^{-\theta h_{k}}, \quad k \geq 1 .
$$

It will be convenient to introduce the notation

$$
\Delta\left(u_{k}\right)=b+\lambda D\left(u_{k}\right)
$$


After rearranging terms, we then obtain

$$
\Delta\left(u_{k}\right) e^{\lambda h_{k}-\delta t_{k}}+\frac{\theta S_{0}}{\beta} e^{\beta t_{k}-\theta h_{k}}=b e^{\lambda h_{k+1}-\delta t_{k+1}}+\frac{\theta S_{0}}{\beta} e^{\beta t_{k+1}-\theta h_{k+1}} e^{\theta u_{k+1}} .
$$

Now, using (12) twice, i.e., stationarity with respect to $t_{k}$ and $t_{k+1}$, we get

$$
\Delta\left(u_{k}\right) e^{\lambda h_{k}-\delta t_{k}}+\frac{\theta \delta}{\beta} \kappa\left(u_{k}\right) e^{\lambda h_{k}-\delta t_{k}}=b e^{\lambda h_{k+1}-\delta t_{k+1}}+\frac{\theta \delta}{\beta} \kappa\left(u_{k+1}\right) e^{\lambda h_{k+1}-\delta t_{k+1}} e^{\theta u_{k+1}}
$$

After multiplying both sides by $e^{-\lambda h_{k}}$, we get

$$
\left(\Delta\left(u_{k}\right)+\frac{\theta \delta}{\beta} \kappa\left(u_{k}\right)\right) e^{-\delta t_{k}}=\left(b+\frac{\theta \delta}{\beta} \kappa\left(u_{k+1}\right) e^{\theta u_{k+1}}\right) e^{\lambda u_{k+1}-\delta t_{k+1}}, \quad k \geq 1 .
$$

In order to eliminate $t_{k}$ (and $t_{k+1}$ ) we introduce parameters $\bar{\delta}$ and $q$ according to

$$
\bar{\delta}:=\frac{\delta}{\beta+\delta}, \quad q:=\bar{\delta}(\lambda+\theta)-\lambda .
$$

Then, we may write, by using (12) once more,

$$
e^{-\delta t_{k}}=\left(e^{(\beta+\delta) t_{k}}\right)^{-\bar{\delta}}=\left(\frac{\delta \kappa\left(u_{k}\right)}{S_{0}} e^{(\theta+\lambda) h_{k}}\right)^{-\bar{\delta}}=\left(\frac{S_{0}}{\delta}\right)^{\bar{\delta}} \kappa\left(u_{k}\right)^{-\bar{\delta}} e^{-(q+\lambda) h_{k}}, \quad k \geq 1
$$

Hence, we obtain

$$
\frac{e^{-\delta t_{k+1}}}{e^{-\delta t_{k}}}=\frac{\left(\frac{S_{0}}{\delta}\right)^{\bar{\delta}} \kappa\left(u_{k+1}\right)^{-\bar{\delta}} e^{-(q+\lambda) h_{k+1}}}{\left(\frac{S_{0}}{\delta}\right)^{\bar{\delta}} \kappa\left(u_{k}\right)^{-\bar{\delta}} e^{-(q+\lambda) h_{k}}}=\frac{\kappa\left(u_{k+1}\right)^{-\bar{\delta}} e^{-(q+\lambda) u_{k+1}}}{\kappa\left(u_{k}\right)^{-\bar{\delta}}} .
$$

Substitution into (15) yields

$$
\kappa\left(u_{k}\right)^{-\bar{\delta}}\left[\Delta\left(u_{k}\right)+\frac{\theta \delta}{\beta} \kappa\left(u_{k}\right)\right]=\kappa\left(u_{k+1}\right)^{-\bar{\delta}} e^{-q u_{k+1}}\left[b+\frac{\theta \delta}{\delta} \kappa\left(u_{k+1}\right) e^{\theta u_{k+1}}\right], \quad k \geq 1
$$

To simplify the notation, we introduce functions $L(x)$ and $R(x)$, with $x>0$, as

$$
\begin{gathered}
L(x):=\Delta(x)+\frac{\theta \delta}{\beta} \kappa(x), \\
R(x):=e^{-q x}\left(b+\frac{\theta \delta}{\beta} \kappa(x) e^{\theta x}\right),
\end{gathered}
$$

as well as functions $\mathcal{L}(x)$ and $\mathcal{R}(x)$ according to

$$
\mathcal{L}(x):=\kappa(x)^{-\bar{\delta}} L(x), \quad \mathcal{R}(x):=\kappa(x)^{-\bar{\delta}} R(x) .
$$


Then, we may rewrite the condition for stationarity with respect to $u_{k}$ in the following compact form:

$$
\mathcal{L}\left(u_{k}\right)=\mathcal{R}\left(u_{k+1}\right), \quad k \geq 1
$$

As will become clear in Section 6, this relation implies that for every entry $u_{k}$ in a stationary sequence $u$ its successor $u_{k+1}$ can attain only two values. Before dealing with this surprising fact, we show in the next section another remarkable result, namely that the objective value $f(u, \tau)$ is completely determined by the value of the function $\mathcal{R}$ in $u_{1}$, the first entry of the sequence $u$.

\section{Objective Value at a Stationary Point}

Taking the sum of all stationarity conditions (5) for $t_{k}(k \geq 1)$, we get

$$
\sum_{k=1}^{\infty} \delta D\left(u_{k}\right) e^{\lambda h_{k}-\delta t_{k}}=\sum_{k=1}^{\infty}\left(S_{0} e^{\beta t_{k}-\theta h_{k-1}}-S_{0} e^{\beta t_{k}-\theta h_{k}}\right) .
$$

This yields a linear relation between $I(u, \tau)$ and $A(u, \tau)$ at a stationary point as follows:

$$
\begin{aligned}
\delta I(u, \tau) & =\sum_{k=1}^{\infty} S_{0} e^{\beta t_{k}-\theta h_{k-1}}-\sum_{k=2}^{\infty} S_{0} e^{\beta t_{k-1}-\theta h_{k-1}} \\
& =S_{0} e^{\beta t_{0}-\theta h_{0}}+\sum_{k=1}^{\infty} S_{0}\left[e^{\beta t_{k}}-e^{\beta t_{k-1}}\right] e^{-\theta h_{k-1}} \\
& =S_{0}+\beta A(u, \tau),
\end{aligned}
$$

where we used $t_{0}=h_{0}=0$. On the other hand, the stationarity condition for $u_{1}$ in (13) yields

$$
\begin{aligned}
b e^{\lambda h_{1}-\delta t_{1}}+\sum_{\ell=1}^{\infty} \lambda D\left(u_{\ell}\right) e^{\lambda h_{\ell}-\delta t_{\ell}} & =\sum_{\ell=2}^{\infty} \frac{\theta S_{0}}{\beta}\left(e^{\beta t_{\ell}}-e^{\beta t_{\ell-1}}\right) e^{-\theta h_{\ell-1}} \\
& =\theta\left(A(u, \tau)-\frac{S_{0}}{\beta} e^{\beta t_{1}-\theta h_{0}}+\frac{S_{0}}{\beta} e^{\beta t_{0}-\theta h_{0}}\right) \\
& =\theta A(u, \tau)-\frac{\theta \delta}{\beta} \kappa\left(u_{1}\right) e^{\lambda h_{1}-\delta t_{1}} e^{\theta u_{1}}+\frac{\theta S_{0}}{\beta}
\end{aligned}
$$

where we used $h_{0}=t_{0}=0$ and $h_{1}=u_{1}$. Thus, we obtain a second linear relation between $I(u, \tau)$ and $A(u, \tau)$, namely,

$$
\begin{aligned}
-\lambda I(u, \tau)+\theta A(u, \tau) & =b e^{\lambda h_{1}-\delta t_{1}}+\frac{\theta \delta}{\beta} \kappa\left(u_{1}\right) e^{\lambda h_{1}-\delta t_{1}} e^{\theta u_{1}}-\frac{\theta S_{0}}{\beta} \\
& =\left(b+\frac{\theta \delta}{\beta} \kappa\left(u_{1}\right) e^{\theta u_{1}}\right) e^{\lambda h_{1}-\delta t_{1}}-\frac{\theta S_{0}}{\beta} \\
& =e^{q u_{1}} R\left(u_{1}\right) e^{\lambda h_{1}-\delta t_{1}}-\frac{\theta S_{0}}{\beta}
\end{aligned}
$$


Using (17), with $k=1$, and also $h_{1}=u_{1}$, we obtain

$$
e^{\lambda h_{1}-\delta t_{1}}=e^{\lambda h_{1}}\left(\frac{S_{0}}{\delta}\right)^{\bar{\delta}} \kappa\left(u_{1}\right)^{-\bar{\delta}} e^{-(q+\lambda) u_{1}}=\left(\frac{S_{0}}{\delta}\right)^{\bar{\delta}} \kappa\left(u_{1}\right)^{-\bar{\delta}} e^{-q u_{1}}
$$

Substituting this, we find

$$
-\lambda I(u, \tau)+\theta A(u, \tau)=\left(\frac{S_{0}}{\delta}\right)^{\bar{\delta}} \mathcal{R}\left(u_{1}\right)-\frac{\theta S_{0}}{\beta} .
$$

As a consequence, we now have the following two linear relations between $I(u, \tau)$ and $A(u, \tau)$ : (23) and (24). The determinant of the coefficient matrix equals $\delta \theta-\lambda \beta$, which by (4) is positive. Hence, the system has a unique solution, namely,

$$
\begin{gathered}
(\theta \delta-\beta \lambda) A(u, \tau)=\lambda S_{0}+\delta\left(\frac{S_{0}}{\delta}\right)^{\bar{\delta}} \mathcal{R}\left(u_{1}\right)-\frac{\delta \theta S_{0}}{\beta} . \\
(\theta \delta-\beta \lambda) I(u, \tau)=\beta\left(\frac{S_{0}}{\delta}\right)^{\bar{\delta}} \mathcal{R}\left(u_{1}\right) .
\end{gathered}
$$

By taking the sum at both sides, we obtain

$$
(\theta \delta-\beta \lambda) f(u, \tau)=(\beta+\delta)\left(\frac{S_{0}}{\delta}\right)^{\bar{\delta}} \mathcal{R}\left(u_{1}\right)-\frac{(\delta \theta-\lambda \beta) S_{0}}{\beta} .
$$

The definition (16) of $q$ implies

$$
q=\bar{\delta}(\lambda+\theta)-\lambda=\delta \frac{(\lambda+\theta)}{\beta+\delta}-\lambda=\frac{\delta(\lambda+\theta)-\lambda(\beta+\delta)}{\beta+\delta}=\frac{\theta \delta-\lambda \beta}{\beta+\delta}
$$

Hence, it follows that at a stationary point the objective value is given by

$$
f(u, \tau)=\frac{1}{q}\left(\frac{S_{0}}{\delta}\right)^{\bar{\delta}} \mathcal{R}\left(u_{1}\right)-\frac{S_{0}}{\beta} .
$$

It comes as a surprise that the objective value at a stationary point is completely determined by $u_{1}$ and is a positive constant times $\mathcal{R}\left(u_{1}\right)$ minus a constant. Hence, it remains to find a stationary point for which $\mathcal{R}\left(u_{1}\right)$ is minimal. As we show in the next section, this is possible thanks to some very nice properties of the functions $\mathcal{L}(x)$ and $\mathcal{R}(x)$.

Before proceeding to the next section, an important observation is that (25) indicates that if $\beta>$ 0 , then $\mathcal{R}\left(u_{1}\right)$ must also be positive, since otherwise one would get a negative value for the total cost $f(u, \tau)$, which would be absurd. Indeed, more generally one has

$$
\beta \mathcal{R}(x)>0, \quad x>0 .
$$

For the proof of this inequality we refer to (52) in Appendix A. 


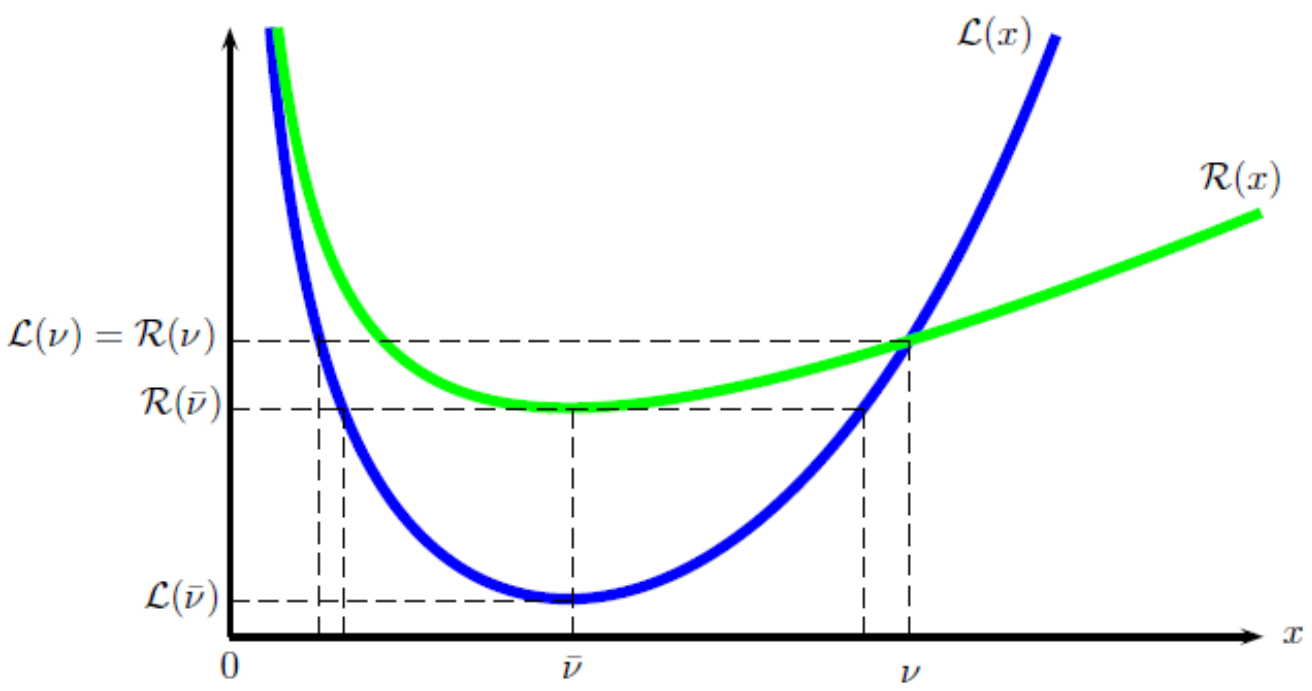

Figure 2. Typical form of the graphs of $\mathcal{L}(x)$ and $\mathcal{R}(x)$

\section{Optimality of the Periodic Solution}

By way of example, Fig. 2 shows the graphs of $\mathcal{L}(x)$ and $\mathcal{R}(x)$ for one of the dike rings. One can show (cf. Appendix A) that these functions always share the following four properties:

Prop. 1: The equation $\mathcal{L}(x)=\mathcal{R}(x)$ has exactly one solution, which is denoted as $v$.

Prop. 2: $\mathcal{L}(x)$ and $\mathcal{R}(x)$ are bounded from below and minimal for the same value $\bar{v}<v$.

Prop. 3: $\mathcal{L}(x)$ and $\mathcal{R}(x)$ are monotonically decreasing for $x<\bar{v}$ and increasing for $x>\bar{v}$.

Prop. 4: One has $\mathcal{L}(x)<\mathcal{R}(x)$ if $x<v$ and $\mathcal{L}(x)>\mathcal{R}(x)$ if $x>v$.

The equation $\mathcal{L}(x)=\mathcal{R}(x)$ is equivalent to $L(x)=R(x)$. Hence, $v$ is defined by

$$
\Delta(v)+\frac{\theta \delta}{\beta} \kappa(v)=e^{-q v}\left(b+\frac{\theta \delta}{\beta} \kappa(v) e^{\theta v}\right), \quad \kappa(v)=\frac{D(v)}{e^{\theta v}-1} .
$$

As Prop. 1 above states, this nonlinear equation determines $v$ uniquely.

The sequence $u=\left(u_{1} ; u_{2} ; u_{3} ; \ldots\right)$ is stationary if and only if $\mathcal{L}\left(u_{k}\right)=\mathcal{R}\left(u_{k+1}\right)$ for each $k \geq 1$. This certainly holds if $u=(v ; v ; v ; \ldots)$. We call this the periodic sequence. It may be understood from Fig. 2 that there exist infinitely many other stationary sequences. Recall from (25) that a stationary sequence $u$ is optimal if and only if $\mathcal{R}\left(u_{1}\right)$ is minimal.

Lemma 6.1. If $u_{k}$ occurs in a stationary sequence, then $\mathcal{L}\left(u_{k}\right) \geq \mathcal{L}(v)$.

Proof. Let $u_{k}$ occur in a stationary sequence. We need to show that $\mathcal{L}\left(u_{k}\right) \geq \mathcal{L}(v)$. Suppose on the contrary that $\mathcal{L}\left(u_{k}\right)<\mathcal{L}(v)$. Since $\mathcal{L}(x)$ is monotonically increasing for $x>\bar{v}$, by Prop. 3, and $v>$ $\bar{v}$, by Prop. 2, we necessarily have $u_{k}<v$. Since $u_{k}$ occurs in a stationary sequence, and its successor $u_{k+1}$ satisfies $\mathcal{R}\left(u_{k+1}\right)=\mathcal{L}\left(u_{k}\right)<\mathcal{L}(v)=\mathcal{R}(v)$, where we used Prop. 1. So, we have $\mathcal{R}\left(u_{k+1}\right)<$ $\mathcal{R}(v)$. For the same reasons as for $\mathcal{L}$, this implies $u_{k+1}<v$. Since $\mathcal{L}(x)<\mathcal{R}(x)$, for all $x<v$, by 
Prop. 4, it follows that $\mathcal{L}\left(u_{k+1}\right)<\mathcal{R}\left(u_{k+1}\right)=\mathcal{L}\left(u_{k}\right)$. Thus, we have shown that $\mathcal{L}\left(u_{k}\right)<\mathcal{L}(v)$ implies $\mathcal{L}\left(u_{k+1}\right)<\mathcal{L}\left(u_{k}\right)$, and as a consequence, also $\mathcal{L}\left(u_{k+1}\right)<\mathcal{L}(v)$. This implies that the $\mathcal{L}$ values of the successors of $u_{k}$ in the sequence $u$ form a strictly decreasing sequence. This sequence is bounded below by $\mathcal{L}(\bar{v})$, by Prop. 2 . Hence, the sequence must converge. A similar argument applies to the sequence of the values $\mathcal{R}\left(u_{k}\right)$. Due to $\mathcal{R}\left(u_{k+1}\right)=\mathcal{L}\left(u_{k}\right)$, for each $k$, the limits of the two sequences must be equal. But this is impossible, since the limit occurs in the interval where $\mathcal{L}(x)<\mathcal{R}(x)$. This contradiction proves the lemma.

Lemma 6.2. If $u_{k}$ occurs in a stationary sequence, then $\mathcal{R}\left(u_{k}\right) \geq \mathcal{R}(v)$.

Proof. If $u_{k} \geq v$, then we have $\mathcal{R}\left(u_{k}\right) \geq \mathcal{R}(v)$, because $\mathcal{R}(x)$ is increasing for $x \geq v$. On the other hand, if $u_{k} \leq v$, then $\mathcal{R}\left(u_{k}\right) \geq \mathcal{L}\left(u_{k}\right)$, by Prop. 3. By Lemma 6.1, we have $\mathcal{L}\left(u_{k}\right) \geq \mathcal{L}(v)$. Hence, $\mathcal{R}\left(u_{k}\right) \geq \mathcal{L}(v)$. Since $\mathcal{L}(v)=\mathcal{R}(v)$, we get $\mathcal{R}\left(u_{k}\right) \geq \mathcal{R}(v)$, as desired.

Theorem 6.3. If $\varrho(v)+(\lambda+\theta) v \geq 0$, then the periodic sequence is optimal.

Proof. Lemma 6.2 implies that $\mathcal{R}\left(u_{1}\right) \geq \mathcal{R}(v)$ for every stationary sequence $u$. According to (25), this implies the theorem, provided that $t_{k} \geq 0$, for $k \geq 1$. Since

$$
t_{k}=\varrho\left(u_{1}\right)+\frac{(\lambda+\theta) h_{k}}{\beta+\delta}=\frac{\varrho(v)}{(\beta+\delta)}+k \frac{\lambda+\theta}{\beta+\delta} \nu, \quad k \geq 1,
$$

for the periodic sequence, this holds only if $\varrho(v)+(\lambda+\theta) v \geq 0$. Hence, the result follows.

It is worth noting that the value of $v$ does not depend on $S_{0}$; neither does the length of the interval between two successive updates. The length of this interval is denoted as $p$, and according to (27), it is given by

$$
p=\frac{\lambda+\theta}{\beta+\delta} \nu
$$

In essence only the moment $t_{1}$ of the first update depends on $S_{0}$, because of its dependence of $\varrho(v)$. Since $\varrho(x)$ is monotonically decreasing with respect to $S_{0}, t_{1}$ decreases when $S_{0}$ increases.

One may easily deduce from (7) and (10), the definitions of $\varrho(x)$ and $\kappa(x)$, respectively, that the condition in Theorem 6.3 holds if and only if

$$
S_{0} \leq \delta \kappa(v) e^{(\lambda+\theta) v}
$$

i.e., if and only if the expected damage costs at $t=0$ do not exceed the threshold value $\delta \kappa e^{(\lambda+\theta) v}$. So, if the value of $t_{1}$ is negative, then it suggests that there is a backlog in the maintenance of the dike. If this happens, we say that the dike is unhealthy; otherwise healthy.

It remains to deal with the unhealthy case. In that case, an immediate heightening of the dike is desirable. So, we then should have $t_{1}=0$. It seems natural to expect that to eliminate the backlog in maintenance a heightening of at least $v$ will be necessary. In the next section, we deal with the question of finding the value of $u_{1}$ that minimizes $f(u, \tau)$. As we will see, it seems impossible to find an analytic solution of this problem. But, we show below that the optimal value of $u_{1}$ in the unhealthy case can be found by minimizing a strictly convex function on a finite interval. 


\section{The Unhealthy Case}

In the unhealthy case the constraint $t_{1} \geq 0$ in (1) is active. This means that at optimality $f(u, \tau)$ is no longer stationary with respect to $t_{1}$. But, we still will have stationarity with respect to $t_{k}$ if $k \geq 2$, and stationarity with respect to $u_{k}$ for all $k \geq 1$. Using this, it is shown in Appendix B that the objective value at a stationary point is given by

$$
f(u, \tau)=D\left(u_{1}\right) e^{\lambda u_{1}}+\frac{e^{-q u_{1}}}{q}\left(\frac{S_{0}}{\delta}\right)^{\bar{\delta}} \mathcal{R}\left(u_{2}\right)-\frac{S_{0}}{\beta} e^{-\theta u_{1}} .
$$

With $u_{1}$ fixed, by using the same arguments as in the healthy case, it follows that $u_{2}=v$ yields the smallest value. Due to (21) and (11), we write

$$
\left(\frac{S_{0}}{\delta}\right)^{\bar{\delta}} \mathcal{R}(v)=\left(\frac{S_{0}}{\delta}\right)^{\bar{\delta}} \kappa(v)^{-\bar{\delta}} R(v)=\left(\frac{\delta}{S_{0}} \kappa(v)\right)^{-\bar{\delta}} R(v)=R(v) e^{-\bar{\delta} \varrho(v)}
$$

Hence, denoting the resulting function of $u_{1}$ as $F\left(u_{1}\right)$, we obtain

$$
F\left(u_{1}\right)=D\left(u_{1}\right) e^{\lambda u_{1}}+\frac{1}{q} R(v) e^{-\bar{\delta} \varrho(v)-q u_{1}}-\frac{S_{0}}{\beta} e^{-\theta u_{1}} .
$$

Remarrk 7.1. If the periodic sequence yields $t_{1}=0$, then the dike is on the boundary of the healthy and unhealthy cases. This happens if and only if $\varrho(v)+(\lambda+\theta) v=0$. Then, the above value must coincide with the objective value of the periodic solution. So, we must have

$$
D(v) e^{\lambda v}+\frac{e^{-q v}}{q}\left(\frac{S_{0}}{\delta}\right)^{\bar{\delta}} \mathcal{R}(v)-\frac{S_{0}}{\beta} e^{-\theta v}=\frac{1}{q}\left(\frac{S_{0}}{\delta}\right)^{\bar{\delta}} \mathcal{R}(v)-\frac{S_{0}}{\beta} .
$$

We leave it to the reader to verify that this equality holds.

It remains to find the value of $u_{1}$ that minimizes $F\left(u_{1}\right)$. In considering this minimization problem, we should respect the conditions (1) for admissibility of the sequence $u=\left(u_{1} ; v ; v ; \ldots\right)$. Since the solution is periodic from $t=t_{2}$ on, (1) boils down to the simple condition $t_{2}>0$. By (9), and since $u_{2}=v$ and $h_{2}=u_{1}+v$, this holds if and only if

$$
\varrho(v)+(\lambda+\theta)\left(u_{1}+v\right)>0
$$

On the other hand, the dike is unhealthy if and only if $\varrho(v)+(\lambda+\theta) v<0$. So, we have

$$
\varrho(v)+(\lambda+\theta) v<0<\varrho(v)+(\lambda+\theta)\left(u_{1}+v\right) .
$$

It will be convenient to introduce the number $u_{0}$ defined by the relation

$$
\varrho(v)+(\lambda+\theta)\left(u_{0}+v\right)=0
$$


Combining this relation with (32) gives $(\lambda+\theta) v<(\lambda+\theta)\left(u_{0}+v\right)<(\lambda+\theta)\left(u_{1}+v\right)$, which implies

$$
0<u_{0}<u_{1}
$$

We derive from (11) and (33) that $u_{0}$ is also uniquely defined by

$$
S_{0} e^{-\theta\left(u_{0}+v\right)}=\delta \kappa(v) e^{\lambda\left(u_{0}+v\right)}
$$

We are now ready to analyze the behavior of $F\left(u_{1}\right)$ and to find its global minimizer, which is denoted as $u_{1}^{*}$. As we just established, we have $u_{1}^{*} \in\left(u_{0}, \infty\right)$. We show below that $u_{1}^{*}$ belongs to the finite interval $\left(u_{0}, u_{0}+v\right]$ and can be found easily. For this, we need a couple of lemmas, which require rather tedious proofs. We also feel free in this section to use some results from Appendix A.

We start with computing the first two derivatives of $F\left(u_{1}\right)$ with respect to $u_{1}$. One has

$$
\begin{aligned}
& F^{\prime}\left(u_{1}\right)=\Delta\left(u_{1}\right) e^{\lambda u_{1}}-R(v) e^{-\bar{\delta} \varrho(v)-q u_{1}}+\theta \frac{S_{0}}{\beta} e^{-\theta u_{1}} \\
& F^{\prime \prime}\left(u_{1}\right)=\lambda\left[b+\Delta\left(u_{1}\right)\right] e^{\lambda u_{1}}+q R(v) e^{-\bar{\delta} \varrho(v)-q u_{1}}-\theta^{2} \frac{S_{0}}{\beta} e^{-\theta u_{1}}
\end{aligned}
$$

Lemma 7.2. $F\left(u_{1}\right)$ is convex for large enough values of $u_{1}$.

Proof. Since $q>0$ and $\theta>0$, the last two terms in the expression for $F^{\prime \prime}\left(u_{1}\right)$ converge to zero if $u_{1}$ grows to infinity. If $\lambda>0$, then the first term grows exponentially fast to infinity. So, if $\lambda>0$, then $F\left(u_{1}\right)$ is convex for large values of $u_{1}$. This also holds if $\lambda=0$, as we now show. We then have

$$
F^{\prime \prime}\left(u_{1}\right)=\left(q e^{-\bar{\delta} \varrho(v)} R(v)-\theta^{2} \frac{S_{0}}{\beta} e^{-r u_{1}}\right) e^{-q u_{1}}
$$

where $r=\theta-q$. Now, $r$ has the same sign as $\beta$, as it follows from

$$
r=\theta-\frac{\theta \delta-\lambda \beta}{\beta+\delta}=\frac{\theta(\beta+\delta)-\theta \delta+\lambda \beta}{\beta+\delta}=\beta \frac{\lambda+\theta}{\beta+\delta} .
$$

This makes clear that if $u_{1}$ grows to infinity, the second term in the bracketed expression converges to zero if $\beta>0$ and to $\infty$ if $\beta<0$. Hence, the bracketed expression goes to $\infty$ if $\beta<0$. If $\beta>0$, then we use (52), which implies that $\beta$ and $R(v)$ have the same sign. Since the bracketed expression converges to $q e^{-\bar{\delta} \varrho(v)} R(v)$ in that case, and $R(v)>0$, we conclude that $F^{\prime \prime}\left(u_{1}\right)>0$ for large values of $u_{1}$. This proves the lemma.

Lemma 7.3. $F\left(u_{1}\right)$ has at most one point of inflection on the interval $(0, \infty)$.

Proof. Recall that $R(v), \beta$ and $r$ are nonzero and have the same sign. Therefore, the expression (37) for $F^{\prime \prime}\left(u_{1}\right)$ has the form of the function $f(x)$ defined by

$$
f(x)=\lambda(A+B x) e^{\lambda x}+C e^{-q x}-D e^{-\theta x},
$$


where $x=u_{1}$ and the parameters $A, B, C$ and $D$ have temporary meanings such that

$$
A \geq 0, \quad B \geq 0, \quad C D>0, \quad r D>0 .
$$

Using $\theta=q+r$, it follows that

$$
\begin{aligned}
f^{\prime}(x) & =\lambda B e^{\lambda x}+\lambda^{2}(A+B x) e^{\lambda x}-q C e^{-q x}+\theta D e^{-\theta x} \\
& =\lambda B e^{\lambda x}+\lambda^{2}(A+B x) e^{\lambda x}-q C e^{-q x}+(q+r) D e^{-\theta x} \\
& =\lambda B e^{\lambda x}+\lambda^{2}(A+B x) e^{\lambda x}-q\left(C e^{-q x}-D e^{-\theta x}\right)+r D e^{\theta x} \\
& =\lambda B e^{\lambda x}+\lambda^{2}(A+B x) e^{\lambda x}-q\left(f(x)-\lambda(A+B x) e^{\lambda x}\right)+r D e^{\theta x} .
\end{aligned}
$$

Assuming $f(x)=0$ we get, for any $x \geq 0$,

$$
f^{\prime}(x)=\lambda B e^{\lambda x}+\lambda^{2}(A+B x) e^{\lambda x}+q \lambda(A+B x) e^{\lambda x}+r D e^{-\theta x} \geq r D e^{-\theta x}>0 .
$$

This proves that $f^{\prime}(x)>0$, whenever $f(x)=0$, which implies that $f(x)$ vanishes for at most one value of $x$. Hence, the lemma follows.

We proceed by showing that $F\left(u_{1}\right)$ is strictly convex at $u_{1}=u_{0}+v$. Before doing this, we deal with three relations that will be useful in the proofs below. The first relation is

$$
q L(v)-\theta \frac{\theta \delta}{\beta} \kappa(v)=q\left(L(v)-\frac{\theta \delta}{\beta} \kappa(v)\right)-r \frac{\theta \delta}{\beta} \kappa(v)=q \Delta(v)-\theta(\lambda+q) \kappa(v),
$$

where we used $\theta=q+r$, the definition of $L(v)$, the definition (16) of $q$ and

$$
\frac{\delta r}{\beta}=\frac{\delta(\theta-q)}{\beta}=\frac{(\delta \theta-\beta \lambda)+\beta \lambda-\delta q}{\beta}=\frac{(\beta+\delta) q+\beta \lambda-\delta q}{\beta}=\lambda+q .
$$

In a similar way, one can show that

$$
q R(v) e^{q v}-\theta \frac{\theta \delta}{\beta} \kappa(v) e^{\theta v}=q b-\theta(\lambda+q) \kappa(v) e^{\theta v}
$$

Finally, as a consequence of the definition (10) of $\kappa(x)$, formula (48) for its derivative and the fact that $e^{\theta x} \kappa(x)=\kappa(x)+D(x)$, we obtain, for any $x>0$,

$$
\kappa^{\prime}(x)+(\lambda+\theta) \kappa(x)=\frac{\Delta(x)-\theta \kappa(x)}{e^{\theta x}-1} .
$$

Lemma 7.4. $F\left(u_{1}\right)$ is strictly convex at $u_{1}=u_{0}+v$.

Proof. Substitution of $u_{1}=u_{0}+v$ into (37) yields

$$
F^{\prime \prime}\left(u_{0}+v\right)=\lambda\left(b+\Delta\left(u_{0}+v\right)\right) e^{\lambda\left(u_{0}+v\right)}+q R(v) e^{-\bar{\delta} \varrho(v)-q\left(u_{0}+v\right)}-\theta^{2} \frac{S_{0}}{\beta} e^{-\theta\left(u_{0}+v\right)} .
$$

By using (16) and (33) successively, we obtain 


$$
\begin{aligned}
-\bar{\delta} \varrho(v)-q\left(u_{0}+v\right) & =-\bar{\delta} \varrho(v)+[-\bar{\delta}(\lambda+\theta)+\lambda]\left(u_{0}+v\right) \\
& =-\bar{\delta}\left[\varrho(v)+(\lambda+\theta)\left(u_{0}+v\right)\right]+\lambda\left(u_{0}+v\right) \\
& =\lambda\left(u_{0}+v\right) .
\end{aligned}
$$

Using this, $R(v)=L(v),(35)$ and (38), we get

$$
\begin{aligned}
F^{\prime \prime}\left(u_{0}+v\right) & =\lambda\left[b+\Delta\left(u_{0}+v\right)\right] e^{\lambda\left(u_{0}+v\right)}+q R(v) e^{\lambda\left(u_{0}+v\right)}-\theta \frac{\theta \delta}{\beta} \kappa(v) e^{\lambda\left(u_{0}+v\right)} \\
& =e^{\lambda\left(u_{0}+v\right)}\left[\lambda\left[b+\Delta\left(u_{0}+v\right)\right]+q L(v)-\theta \frac{\theta \delta}{\beta} \kappa(v)\right] \\
& =e^{\lambda\left(u_{0}+v\right)}\left[\lambda\left[b+\Delta\left(u_{0}+v\right)\right]+q \Delta(v)-\theta(\lambda+q) \kappa(v)\right] .
\end{aligned}
$$

We have $\Delta\left(u_{0}+v\right)=\lambda b u_{0}+\Delta(v) \geq \Delta(v)$. We therefore obtain

$$
e^{-\lambda\left(u_{0}+v\right)} F^{\prime \prime}\left(u_{0}+v\right) \geq \lambda b+(\lambda+q) \Delta(v)-\theta(\lambda+q) \kappa(v) .
$$

So, it suffices for the proof to show $g(x)>0$ at $x=v$, where

$$
g(x):=\lambda b+(\lambda+q)[\Delta(x)-\theta \kappa(x)]
$$

Recall from Lemma A.1 that $\kappa(x)$ is monotonically decreasing. Since $\Delta(x)$ is nondecreasing, it follows that $g(x)$ is monotonically increasing. Therefore, since $\bar{v}<v$, it suffices to show that $g(\bar{v}) \geq$ 0 . By definition, $\bar{v}$ is the (unique) solution of (50). This implies

$$
\Delta(\bar{v})-\theta \kappa(\bar{v})=\frac{\lambda b \kappa(\bar{v})}{\bar{\delta} \kappa^{\prime}(\bar{v})} \leq 0
$$

the last inequality holds because $\lambda b \geq 0, \bar{\delta}>0, \kappa(x)>0$ and $\kappa^{\prime}(x)<0$, for $x>0$, by Lemma A.1. Hence we get, by using $\lambda+q=\bar{\delta}(\lambda+\theta)$, once more,

$$
g(\bar{v})=\lambda b+\bar{\delta}(\lambda+\theta) \frac{\lambda b \kappa(\bar{v})}{\bar{\delta} \kappa^{\prime}(\bar{v})}=\frac{\lambda b}{\kappa^{\prime}(\bar{v})}\left[\kappa^{\prime}(\bar{v})+(\lambda+\theta) \kappa(\bar{v})\right]
$$

The first factor is negative or zero. For the second factor, we get, by using (40) with $x=\bar{v}$,

$$
\kappa^{\prime}(\bar{v})+(\lambda+\theta) \kappa(\bar{v})=\frac{\Delta(\bar{v})-\theta \kappa(\bar{v})}{e^{\theta \bar{v}}-1} \leq 0
$$

due to (42). Thus, we obtain $g(\bar{v}) \geq 0$, which completes the proof.

Now that we know that $F\left(u_{1}\right)$ is convex at $u_{1}=u_{0}+v$, we may conclude from Lemma 7.3 and Lemma 7.2 that $F\left(u_{1}\right)$ is convex for all $u_{1} \geq u_{0}+v$. This holds because otherwise there would exist at least two points of inflection, which contradicts Lemma 7.3. So, if there exists an inflection point $\tilde{u}$, then $\tilde{u}<u_{0}+v$ and $F\left(u_{1}\right)$ is concave for $u_{1}<\tilde{u}$.

Next, we establish that $u_{0}+v$ is also an upper bound for $u_{1}^{*}$.

Lemma 7.5. If $\lambda b>0$, then $F^{\prime}\left(u_{0}+v\right)>0$. Otherwise, if $\lambda b=0$, then $F^{\prime}\left(u_{0}+v\right)=0$. 
Proof. One has

$$
F^{\prime}\left(u_{0}+v\right)=\Delta\left(u_{0}+v\right) e^{\lambda\left(u_{0}+v\right)}-R(v) e^{-\bar{\delta} \varrho(v)} e^{-q\left(u_{0}+v\right)}+\theta \frac{S_{0}}{\beta} e^{-\theta\left(u_{0}+v\right)} .
$$

Using successively (41), (35) and (19), we get

$$
\begin{aligned}
F^{\prime}\left(u_{0}+v\right) & =\Delta\left(u_{0}+v\right) e^{\lambda\left(u_{0}+v\right)}-R(v) e^{-\lambda\left(u_{0}+v\right)}+\frac{\theta \delta}{\beta} \kappa(v) e^{\lambda\left(v+u_{0}\right)} \\
& =\left[\Delta\left(u_{0}+v\right)-L(v)+\frac{\theta \delta}{\beta} \kappa(v) e^{\lambda\left(u_{0}+v\right)}\right. \\
& =\left[\Delta\left(u_{0}+v\right)-\Delta(v)\right] e^{\lambda\left(u_{0}+v\right)} \\
& =\lambda b u_{0} e^{\lambda\left(u_{0}+v\right)}
\end{aligned}
$$

Since $\lambda \geq 0, b \geq 0$, and $u_{0}>0$, this proof of the lemma is complete.

We now have that $F\left(u_{1}\right)$ is convex for $u_{1} \geq u_{0}+v$ and its derivative at $u_{0}+v$ is nonnegative. This implies that $F\left(u_{1}\right)$ is monotonically increasing for $u_{1} \geq u_{0}+v$. Therefore, the minimizer $u_{1}^{*}$ satisfies $u_{1}^{*} \leq u_{0}+v$. Hence, $u_{1}^{*} \in\left(u_{0}, u_{0}+v\right]$.

Before proceeding, we use Fig. 3 to illustrate the current situation. This figure shows the graph of $F\left(u_{1}\right)$, for $u_{1} \geq u_{0}$, for one of the dikes in The Netherlands. Note that the graph has a point of inflection $\tilde{u}$. As a consequence of Lemma 7.3, there are no more points of inflection. Hence, $F\left(u_{1}\right)$ is concave for all $u_{1}<\tilde{u}$ and therefore also for $u_{1}=u_{0}$. In this figure, $u_{0}$ is a local minimizer of $F\left(u_{0}\right)$, but not a global minimizer. The question arises whether $u_{0}$ can also be a global minimizer. In that case, we would get $u_{1}^{*}=u_{0}$, which would be in conflict with (33).

To deal with the above question, let us assume for the moment that $u_{1}^{*}=u_{0}$. Then, we get

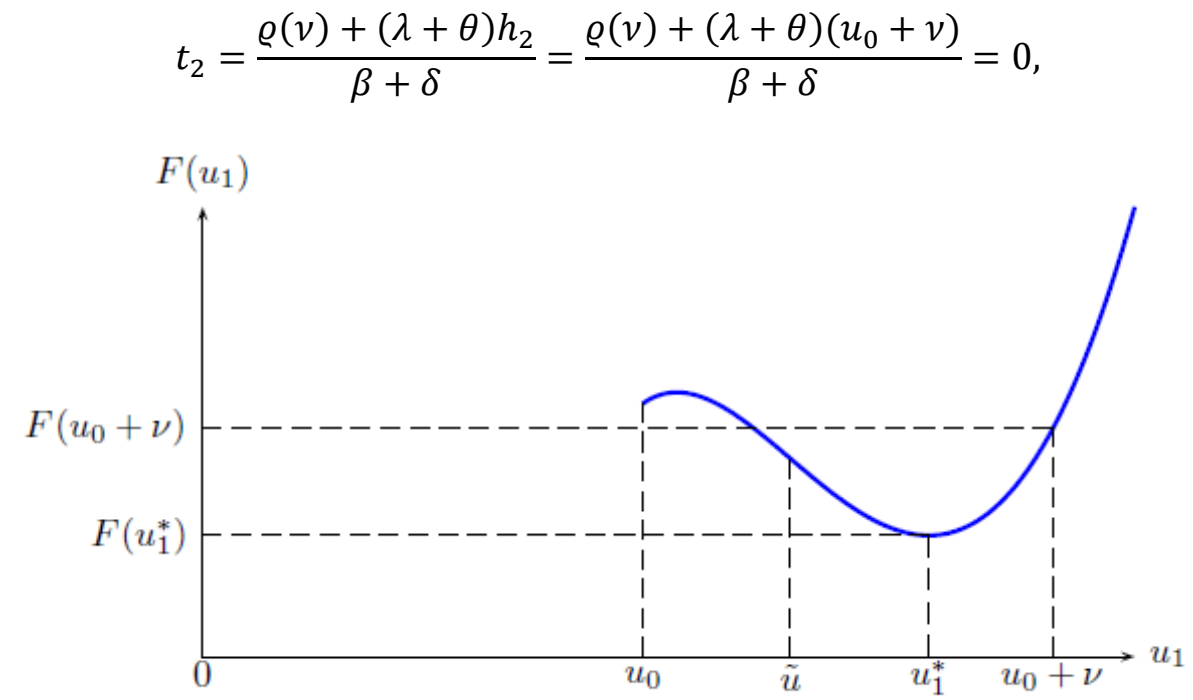

Figure 3. Typical graph of $F\left(u_{1}\right), u_{1}>u_{0}$ 
where the last equality is due to the definition (33) of $u_{0}$. But, this means that the upgrades with $u_{0}$ at $t_{1}$ and with $v$ at $t_{2}$ take place at the same moment $t=0$. According to (3), the corresponding costs are

$$
D\left(u_{0}\right) e^{\lambda h_{1}}+D(v) e^{\lambda h_{2}}=\left(c+b u_{0}\right) e^{\lambda u_{0}}+(c+b v) e^{\lambda\left(u_{0}+v\right)} .
$$

The same situation arises by performing one upgrade of size $u_{0}+v$ at $t=0$, whose costs are

$$
D\left(u_{0}+v\right) e^{\lambda h_{2}}=\left(c+b\left(u_{0}+v\right)\right) e^{\lambda\left(u_{0}+v\right)} .
$$

From an engineering point of view, it seems realistic to assume that the 'one-step' strategy is cheaper than the first strategy, which splits the upgrade in two upgrades. One has

$$
\begin{aligned}
D\left(u_{0}\right) e^{\lambda h_{1}}+D(v) e^{\lambda h_{2}}-D\left(u_{0}+v\right) e^{\lambda h_{2}} & =D\left(u_{0}\right) e^{\lambda u_{0}}-b u_{0} e^{\lambda\left(u_{0}+v\right)} \\
& =e^{\lambda u_{0}}\left(c+b u_{0}\right)-b u_{0} e^{\lambda v} \\
& =e^{\lambda u_{0}}\left(c-b u_{0}\left(e^{\lambda v}-1\right)\right) .
\end{aligned}
$$

The last expression is positive if and only if

$$
c>b u_{0}\left(e^{\lambda v}-1\right)=b\left(e^{\lambda v}-1\right)
$$

In the sequel, we assume that this condition is satisfied. As the next lemma shows, this assumption resolves the aforementioned conflict with (33). It guarantees that $u_{0}$ is not a global minimizer of $F\left(u_{1}\right)$.

Lemma 7.6. $F\left(u_{0}\right)=\left[D\left(u_{0}\right) e^{\lambda u_{0}}-b u_{0} e^{\lambda\left(u_{0}+v\right)}\right]+F\left(u_{0}+v\right)$.

Proof. One has

$$
\begin{gathered}
F\left(u_{0}\right)=D\left(u_{0}\right) e^{\lambda u_{0}}+\frac{1}{q} R(v) e^{-\bar{\delta} \varrho(v)-q u_{0}}-\frac{S_{0}}{\beta} e^{-\theta u_{0}} \\
F\left(u_{0}+v\right)=D\left(u_{0}+v\right) e^{\lambda\left(u_{0}+v\right)}+\frac{1}{q} R(v) e^{-\bar{\delta} \varrho(v)-q\left(u_{0}+v\right)}-\frac{S_{0}}{\beta} e^{-\theta\left(u_{0}+v\right)} .
\end{gathered}
$$

By using (41) and (35), we may therefore write

$$
F\left(u_{0}\right)-D\left(u_{0}\right) e^{\lambda\left(u_{0}\right)}=\frac{1}{q} R(v) e^{\lambda\left(u_{0}+v\right)+q v}-\frac{\delta}{\beta} \kappa(v) e^{(\lambda+\theta) v+\lambda u_{0}},
$$

and, by also using $D\left(u_{0}+v\right)=b u_{0}+D(v)$, we have

$$
F\left(u_{0}+v\right)-b u_{0} e^{\lambda\left(u_{0}+v\right)}=D(v) e^{\lambda\left(u_{0}+v\right)}+\frac{1}{q} R(v) e^{\lambda\left(u_{0}+v\right)}-\frac{\delta}{\beta} \kappa(v) e^{\lambda\left(v+u_{0}\right)} .
$$

The lemma will follow if the right-hand side members in the above equations are equal. After dividing by the common factor $e^{\lambda\left(u_{0}+v\right)}$, this leads to the following equation: 


$$
\frac{1}{q} R(v) e^{q v}-\frac{\delta}{\beta} \kappa(v) e^{\theta v}=D(v)+\frac{1}{q} R(v)-\frac{\delta}{\beta} \kappa(v)
$$

Since $\kappa(v) e^{\theta v}=\kappa(v)+D(v)$, this further reduces to

$$
\frac{1}{q} R(v) e^{q v}-\frac{\delta}{\beta} D(v)=D(v)+\frac{1}{q} R(v)
$$

After multiplication of both sides with $q$, we get the equivalent equality,

$$
R(v) e^{q v}-\frac{q \beta+\delta}{\beta} D(v)=R(v)
$$

Using the definitions of $q$ and $R(x)$, the left-hand side expression reduces to $L(v)$, as follows:

$$
\begin{aligned}
e^{q v} R(v)-q \frac{\beta+\delta}{\beta} D(v) & =e^{q v} R(v)-\frac{\theta \delta-\lambda \beta}{\beta} D(v) \\
& =b+\frac{\theta \delta}{\beta} \kappa(v) e^{\theta v}-\frac{\theta \delta}{\beta} D(v)+\lambda D(v) \\
& =\Delta(v)+\frac{\theta \delta}{\beta}\left[\kappa(v) e^{\theta v}-D(v)\right] \\
& =\Delta(v)+\frac{\theta \delta}{\beta} \kappa(v)=L(v) .
\end{aligned}
$$

Since $L(v)=R(v)$, by the definition of $v$, the lemma follows.

Since the bracketed expression in the lemma is a positive multiple of $c-b u_{0}\left(e^{\lambda v-1}\right)$, by (43), we conclude that $u_{1}^{*} \in\left(u_{0}, u_{0}+v\right]$. So $u_{1}$ can be obtained by minimizing a convex function on the finite interval $\left(\tilde{u}, u_{0}+v\right]$ if there is a point of inflection $\tilde{u}>u_{0}$ and on the finite interval $\left(u_{0}, u_{0}+v\right]$ if there is no point of inflection.

Thus, we have shown that in the unhealthy case the minimizer $u_{1}^{*}$ of $f(u, \tau)=F\left(u_{1}\right)$ can be found by minimizing a convex function on a finite interval. In cases where $\lambda b=0$, we know at forehand that $u_{1}^{*}=u_{0}+v$, because then $F^{\prime}\left(u_{0}+v\right)=0$, by Lemma 7.5. Moreover, the solution is periodic from $t=t_{2}$ on, where

$$
t_{2}=\frac{\varrho(v)+(\lambda+\theta)\left(u_{1}^{*}+v\right)}{\beta+\delta}
$$

After $t=t_{2}$, the solution is independent of $S_{0}$ : all subsequent upgrades have the same value $v$ and occur periodically; the interval between two subsequent upgrades is $p$, as given by (28).

It may be mentioned that for the 22 dikes for which the data are available (cf. Appendix C), there is always an inflection point $\tilde{u}>u_{0}$. Note that this holds if and only if $F^{\prime \prime}\left(u_{0}\right)<0$. We would have liked to prove this in the general case, under the condition (44), but we did not succeed. We leave this as a challenge to the reader. 


\section{Computational Results}

We applied our results to the 22 dikes whose data are given in Table 2 in Appendix C. The resulting solutions for these dikes are presented in Table 1.

The first column in this table uses a parameter $\sigma$, which is defined by

$$
\sigma=\frac{S_{0}}{\delta \kappa(v) e^{(\lambda+\theta) v}}
$$

where the denominator is the threshold value for $S_{0}$ in (29), that separates healthy dikes $(\sigma \leq 1)$ from unhealthy dikes $(\sigma>1)$. As one might expect this separation is also clear from the values of $t_{1}$ and $u_{1}$ in the table: for the unhealthy dikes, one has $t_{1}=0$ and $u_{1}>v$, whereas for healthy dikes, $t_{1} \geq 0$ and $u_{1}=v$.

The second column in the table serves to show that all dikes satisfy the condition (44). This condition can be reformulated in terms of an upper $\bar{\sigma}$ bound for $\sigma$. This will be explained now. First, we consider the following lemma.

Lemma 8.1. With $\sigma$ as just defined, one has

$$
u_{0}=\frac{\log \sigma}{\lambda+\theta}
$$

Proof. Due to (45), we may write

$$
(\lambda+\theta) v=\log \frac{S_{0}}{\sigma \delta \kappa(v)}=-\log \frac{\sigma \delta \kappa(v)}{S_{0}}=-\varrho(v)-\log \sigma
$$

Due to the definition (33) of $u_{0}$, this implies $(\lambda+\theta) u_{0}=\log \sigma$, whence we obtain (46).

Due to the above lemma, condition (44) can be written as

$$
c>b \frac{\log \sigma}{\lambda+\theta}\left(e^{\lambda v}-1\right)
$$

Since $c>0$, this certainly holds if $\lambda b=0$. If $\lambda b>0$, however, it puts an upper bound on the value of $\sigma$, namely,

$$
\log \sigma<\frac{(\lambda+\theta) c}{b\left(e^{\lambda v}-1\right)}
$$

The second column in the table shows the resulting upper bound for $\sigma$, which is denoted as $\bar{\sigma}$. The table not only makes clear that $\sigma<\bar{\sigma}$, so that (44) holds for all dikes, but also that $\bar{\sigma}$ is very large for some dikes. 
Table 1. Solutions for the 22 dikes as specified in Table 2 in Appendix C.

\begin{tabular}{rrrrrrrrr}
\hline no. & $\sigma$ & $\bar{\sigma}$ & $v$ & $u_{1}$ & $p$ & $t_{1}$ & $t_{2}$ & $f\left(u^{*}, \tau^{*}\right)$ \\
\hline 10 & 0.2466 & $1.90 \mathrm{e}+04$ & 56.96 & 57.12 & 56.96 & 45.80 & 102.92 & 40.04 \\
11 & 0.2771 & $\infty$ & 62.42 & 58.89 & 62.42 & 42.44 & 101.33 & 110.23 \\
15 & 1.1849 & $9.35 \mathrm{e}+03$ & 53.29 & 51.54 & 55.96 & 0.00 & 51.20 & 545.18 \\
16 & 0.8001 & $1.78 \mathrm{e}+06$ & 52.59 & 54.04 & 52.59 & 3.50 & 57.54 & 1089.68 \\
22 & 0.4463 & $2.91 \mathrm{e}+12$ & 53.70 & 62.43 & 53.70 & 12.72 & 75.16 & 309.25 \\
23 & 0.297 & $6.36 \mathrm{e}+05$ & 55.24 & 48.24 & 55.24 & 56.06 & 104.30 & 20.07 \\
24 & 0.5871 & $1.08 \mathrm{e}+03$ & 61.81 & 42.80 & 61.81 & 8.01 & 50.80 & 297.26 \\
35 & 1.2344 & $7.06 \mathrm{e}+01$ & 59.65 & 41.73 & 63.89 & 0.00 & 41.08 & 345.23 \\
38 & 1.1016 & $2.24 \mathrm{e}+01$ & 62.04 & 51.31 & 65.27 & 0.00 & 50.80 & 172.07 \\
40 & 0.0615 & $4.87 \mathrm{e}+02$ & 78.80 & 61.68 & 78.80 & 90.90 & 152.57 & 3.83 \\
41 & 2.1307 & $3.61 \mathrm{e}+02$ & 74.66 & 62.95 & 100.55 & 0.00 & 60.13 & 325.89 \\
42 & 0.6144 & $5.50 \mathrm{e}+03$ & 72.24 & 61.43 & 72.24 & 15.43 & 76.86 & 79.24 \\
43 & 0.7688 & $3.58 \mathrm{e}+02$ & 43.49 & 64.66 & 73.49 & 8.39 & 73.05 & 1304.79 \\
44 & 2.9440 & $3.71 \mathrm{e}+01$ & 49.54 & 55.42 & 76.91 & 0.00 & 50.06 & 206.50 \\
45 & 2.5159 & $1.70 \mathrm{e}+01$ & 41.49 & 50.94 & 61.80 & 0.00 & 45.69 & 33.72 \\
47 & 1.3608 & $9.51 \mathrm{e}+01$ & 55.60 & 51.87 & 65.26 & 0.00 & 50.74 & 64.10 \\
48 & 1.2888 & $5.68 \mathrm{e}+00$ & 50.84 & 42.47 & 58.24 & 0.00 & 40.57 & 403.00 \\
49 & 0.3808 & $4.41 \mathrm{e}+01$ & 45.65 & 52.97 & 45.65 & 31.66 & 84.62 & 74.04 \\
50 & 2.6718 & $\infty$ & 61.97 & 58.78 & 95.87 & 0.00 & 58.78 & 53.47 \\
51 & 0.3372 & $1.86 \mathrm{e}+01$ & 40.49 & 51.49 & 40.49 & 35.48 & 56.97 & 54.18 \\
52 & 0.8966 & $1.48 \mathrm{e}+02$ & 45.74 & 57.79 & 45.74 & 3.52 & 61.31 & 245.38 \\
53 & 2.0317 & $1.12 \mathrm{e}+04$ & 66.13 & 68.54 & 86.57 & 0.00 & 66.58 & 307.48 \\
\hline
\end{tabular}

If (47) does not hold, then we call a dike very unhealthy, because then splitting an upgrade of the dike at some moment in two steps (at the same time) may become more advantageous than doing it in one step. For such dikes, the solution of our model may yield $t_{1}=t_{2}=0$. As we made it clear, this is in conflict with the assumptions underlying the model. So, we must conclude that the model breaks down in such cases.

Finally, it may be worth to emphasize that at the end of Section 6 we gave a 'physical' proof for the inequality $u_{1}>v$ in the unhealthy case. It is clear from Table 1 that this inequality holds indeed. Unfortunately, we were not able to prove this inequality mathematically. We leave it as a challenge for the reader.

\section{Acknowledgement}

Thanks are due to my co-authors in $[1,2,3]$ for some useful discussions on details of this paper.

\section{References}

[1] Brekelmans, R.C.M., den Hertog, D., Roos, C., and Eijgenraam, C.J.J. (2012), Safe dike heights at minimal costs: The nonhomogeneous case, Operations Research, 60(6), 13421355 .

[2] Eijgenraam, C.J.J., Brekelmans, R.C.M., den Hertog, D., and Roos, C. (2016), Optimal strategies for flood prevention, Management Science, 63(5), 1-15. DOI $10.1287 /$ mnsc.2015.2395. 
[3] Eijgenraam, C., Kind, J., Bak, C., Brekelmans, R., den Hertog, D., Duits, M., Roos, C., Vermeer, P., and Kuijken, W. (2014), Economically efficient standards to protect the Netherlands against flooding, Interfaces, 44(1), 7-21.

[4] Eijgenraam, C.J.J. (2006), Optimal safety standards for dike-ring areas. CPB Discussion Paper 62, Netherlands Bureau for Economic Policy Analysis, The Hague, March 2006.

[5] Eijgenraam, C.J.J. (2007), Oplossing van het veiligheidsprobleem bij meer dan één dijkvak per dijkring. CPB Memorandum 5/2007/01, Netherlands Bureau for Economic Policy Analysis, The Hague, February 2007 (in Dutch).

[6] van Dantzig, D. (1956), Economic decision problems for flood prevention, Econometrica, 24(3), 276-287. 


\section{Appendix A. Proofs of Properties of $\mathcal{L}(x)$ and $\mathcal{R}(x)$}

Recall that $D(x)=c+b x$ and that $\kappa(x)$ as defined in (10). Below, we frequently use the first and second derivatives of $\kappa(x)$, which are given by

$$
\begin{gathered}
\kappa^{\prime}(x)=\frac{b-\theta e^{\theta x} \kappa(x)}{e^{\theta x}-1} \\
\kappa^{\prime \prime}(x)=-\frac{\theta e^{\theta x}}{e^{\theta x}-1}\left(\theta \kappa(x)+2 \kappa^{\prime}(x)\right) .
\end{gathered}
$$

Lemma A.1. $\kappa(x)$ is strictly convex and monotonically decreasing to zero.

Proof. From (48) it is clear that $\kappa^{\prime}(x)$ has the same sign as

$$
b\left(e^{\theta x}-1\right)-\theta e^{\theta x} D(x) .
$$

The value of this expression at $x=0$ is $-\theta c$, which is negative, and its derivative is

$$
b \theta e^{\theta x}-\theta^{2} e^{\theta x} D(x)-\theta e^{\theta x} b=-\theta^{2} e^{\theta x} D(x)<0 .
$$

Therefore, $\kappa^{\prime}(x)$ is negative. Hence $\kappa(x)$ is monotonically decreasing, as stated in the lemma. Moreover, the definition of $\kappa(x)$ makes clear that if $x$ grows, then the limiting value is zero.

Concerning the second the derivative of $\kappa(x)$, we deduce from (49) that it has the same sign as $-2 \kappa^{\prime}(x)-\theta \kappa(x)$, for which we have

$$
-2 \kappa^{\prime}(x)-\theta \kappa(x)=-\frac{2\left(b-\theta e^{\theta x} \kappa(x)\right)+\left(e^{\theta x}-1\right) \theta \kappa(x)}{e^{\theta x}-1}=\frac{\theta e^{\theta x} \kappa(x)+\theta \kappa(x)-2 b}{e^{\theta x}-1} .
$$

Multiplication with $\left(e^{\theta x}-1\right)^{2}$ yields the expression

$$
\theta e^{\theta x} D(x)+\theta D(x)-2\left(e^{\theta x}-1\right) b .
$$

Since $c>0$, this expression is positive if $b=0$, whereas its derivative with respect to $b$ equals

$$
\theta x e^{\theta x}+\theta x-2\left(e^{\theta x}-1\right)
$$

Putting $y:=\theta x$, one may easily verify that $y e^{y}+y-2\left(e^{y}-1\right) \geq 0$, for $y \geq 0$. It thus follows that $\kappa^{\prime \prime}(x)>0$, for all $x>0$, which means that $\kappa(x)$ is strictly convex. Hence, the proof is complete.

We proceed by showing that the derivatives of $\mathcal{L}(x)$ and $\mathcal{R}(x)$ are closely related. 
Lemma A.2. ${ }^{2}$ One has

$$
\mathcal{L}^{\prime}(x)=e^{q x} \mathcal{R}^{\prime}(x)=\kappa(x)^{-\bar{\delta}-1}\left[\lambda b \kappa(x)+\bar{\beta}[\theta \kappa(x)-\Delta(x)] \kappa^{\prime}(x) .\right.
$$

Proof. We start by computing the derivative of $\mathcal{L}(x)$ :

$$
\begin{aligned}
\mathcal{L}^{\prime}(x) & =-\bar{\delta} \kappa(x)^{-\bar{\delta}-1} \kappa^{\prime}(x) L(x)+\kappa(x)^{-\bar{\delta}} L^{\prime}(x)=\kappa(x)^{-\bar{\delta}-1}\left[-\bar{\delta} \kappa^{\prime}(x) L(x)+\kappa(x) L^{\prime}(x)\right] \\
& =\kappa(x)^{-\bar{\delta}-1}\left[-\bar{\delta} \kappa^{\prime}(x)\left(\Delta(x)+\frac{\theta \delta}{\beta} \kappa(x)\right)+\kappa(x)\left(\lambda b+\frac{\theta \delta}{\beta} \kappa^{\prime}(x)\right)\right] .
\end{aligned}
$$

The coefficient of $\kappa(x) \kappa^{\prime}(x)$ in the bracketed expression equals

$$
-\bar{\delta} \frac{\theta \delta}{\beta}+\frac{\theta \delta}{\beta}=(1-\bar{\delta}) \frac{\theta \delta}{\beta}=\left(1-\frac{\delta}{\beta+\delta}\right) \frac{\theta \delta}{\beta}=\frac{\beta}{\beta+\delta} \frac{\theta \delta}{\beta}=\theta \bar{\delta}
$$

Thus, we get

$$
\mathcal{L}^{\prime}(x)=\kappa(x)^{-\bar{\delta}-1}\left[\lambda b \kappa(x)+\bar{\delta} \kappa^{\prime}(x)[\theta \kappa(x)-\Delta(x)]\right]
$$

Hence, the expression for the derivative of $\mathcal{L}(x)$ in the lemma is correct. The derivative of $\mathcal{R}(x)$ is

$$
\begin{aligned}
\mathcal{R}^{\prime}(x) & =-\bar{\delta} \kappa(x)^{-\bar{\delta}-1} \kappa^{\prime}(x) R(x)+\kappa(x)^{-\bar{\delta}} R^{\prime}(x)=\kappa(x)^{-\bar{\delta}-1}\left[-\bar{\delta} \kappa^{\prime}(x) R(x)+\kappa(x) R^{\prime}(x)\right] \\
= & \kappa(x)^{-\bar{\delta}-1}\left[-\bar{\delta} \kappa^{\prime}(x)\left(b e^{-q x}+\frac{\theta \delta}{\beta} \kappa(x) e^{r x}\right)+\kappa(x)\left(-q b e^{-q x}+\frac{\theta \delta}{\beta}\left[\kappa^{\prime}(x)+r \kappa(x)\right] e^{r x}\right)\right] .
\end{aligned}
$$

Hence, the lemma will follow if

$$
\begin{aligned}
\lambda b \kappa(x)+\bar{\delta} \kappa^{\prime}(x) & {[\theta \kappa(x)-\Delta(x)] } \\
= & -\bar{\delta} \kappa^{\prime}(x)\left(b+\frac{\theta \delta}{\beta} \kappa(x) e^{\theta x}\right)+\kappa(x)\left(-q b+\frac{\theta \delta}{\beta}\left[\kappa^{\prime}(x)+r \kappa(x)\right] e^{\theta x}\right) .
\end{aligned}
$$

The coefficient of $\kappa^{\prime}(x) \kappa(x)$ in the left-hand side is $\bar{\delta} \theta$ and the coefficient in the right-hand side:

$$
-\bar{\delta} \frac{\theta \delta}{\beta} e^{\theta x}+\frac{\theta \delta}{\beta} e^{\theta x}=(1-\bar{\delta}) \frac{\theta \delta}{\beta} e^{\theta x}=\bar{\delta} \theta e^{\theta x},
$$

where we used $(1-\bar{\delta}) \delta=\beta \bar{\delta}$. Hence, since $\frac{\theta r}{\beta}=\lambda+q$, it remains to show to show that

$$
(\lambda+q) b \kappa(x)+\bar{\delta} \theta\left(1-e^{\theta x}\right) \kappa^{\prime}(x) \kappa(x)-\bar{\delta} \kappa^{\prime}(x)(\Delta(x)-b)=\theta(\lambda+q) \kappa(x)^{2} e^{\theta x} .
$$

Since $\lambda+q=\bar{\delta}(\lambda+\theta)$ and $\Delta(x)=b+\lambda D(x)$, after dividing by the common factor $\bar{\delta}$ we get:

$$
(\lambda+\theta) b \kappa(x)+\left(1-e^{\theta} x\right) \theta \kappa^{\prime}(x) \kappa(x)-\lambda \kappa^{\prime}(x) D(x)=\theta(\lambda+\theta) \kappa(x)^{2} e^{\theta x} .
$$

\footnotetext{
${ }^{2}$ If $\beta=0$ then, the derivatives of $\mathcal{L}(x)$ and $\mathcal{R}(x)$ are also as given by this lemma, with $q=\theta$ and $\bar{\delta}=1$. This implies that all subsequent results are also valid if $\beta=0$.
} 
Moving the first term at the left to the right and using the expression (48) for $\kappa^{\prime}(x)$, we get

$$
\begin{aligned}
\left(1-e^{\theta x}\right) \theta \kappa^{\prime}(x) \kappa(x)-\lambda \kappa^{\prime}(x) D(x) & =(\lambda+\theta) \kappa(x)\left(\theta \kappa(x) e^{\theta x}-b\right) \\
& =(\lambda+\theta) \kappa(x)\left(1-e^{\theta x}\right) \kappa^{\prime}(x),
\end{aligned}
$$

which reduces to

$$
-\lambda \kappa^{\prime}(x) D(x)=\lambda \kappa(x)\left(1-e^{\theta x}\right) \kappa^{\prime}(x)
$$

Since $-D(x)=\kappa(x)\left(1-e^{\theta x}\right)$, this relation holds true. Hence, the proof is complete.

Lemma A.2 implies that $\mathcal{L}^{\prime}(x)=0$ holds if and only if $\mathcal{R}^{\prime}(x)=0$ and this happens if and only if

$$
\lambda b \kappa(x)+\bar{\delta}[\theta \kappa(x)-\Delta(x)] \kappa^{\prime}(x)=0 .
$$

We claim that this equation has exactly one solution. This is the content of the following lemma.

Lemma A.3. The equation (50) has exactly one solution.

Proof. Multiplying the left-hand side of (50) with $e^{\theta x}-1>0$, we get the function $k(x)$ defined by

$$
k(x):=\lambda b D(x)+\bar{\delta}\left[\theta D(x)-\left(e^{\theta x}-1\right) \Delta(x)\right] \kappa^{\prime}(x)
$$

Now, it suffices for the proof to show that the equation $k(x)=0$ has exactly one solution. We first show that $k(x)$ approaches $-\infty$ if $x$ goes to zero and $k(x)$ converges either to $\infty$ or to a positive number if $x$ goes to $\infty$.

This implies that $k(x)$ attains the value zero for some finite value. From this point on, we assume that $\bar{v}>0$ is such that $k(\bar{v})=0$. Next, we show that $k(x)=0$ implies $k^{\prime}(x)>0$, which implies that $\bar{v}$ is unique.

One has

$$
\lim _{x \downarrow 0} k(x)=\lim _{x \downarrow 0} \lambda b D(0)+\bar{\delta} \theta D(0) \frac{b-\theta \frac{D(0)}{\theta x}}{\theta x}=\lim _{x \downarrow 0} \bar{\delta} \frac{D(0)^{2}}{x^{2}}=-\infty,
$$

where we used $D(0)=c>0$. We next consider the behavior of $k(x)$ if $x$ grows to infinity. We first consider the case where $\lambda=0$. Then, using $D(x)=c+b x$ and $\Delta(x)=b+\lambda D(x)>0$, due to (14), we have

$$
\begin{aligned}
\lim _{x \rightarrow \infty} k(x) & =\lim _{x \rightarrow \infty} \bar{\delta}\left[\theta b x-e^{\theta x} b\right] \frac{b-\theta e^{\theta x} \frac{b x}{e^{\theta x}}}{e^{\theta x}}=\lim _{x \rightarrow \infty} \bar{\delta}\left[-e^{\theta x} b\right] \frac{b-\theta b x}{e^{\theta x}} . \\
& =\lim _{x \rightarrow \infty} \bar{\delta} b^{2}(\theta x-1)=\infty
\end{aligned}
$$

Now, let $\lambda>0$. We then need to distinguish between $b>0$ and $b=0$. If $b>0$, then 


$$
\begin{aligned}
\lim _{x \rightarrow \infty} k(x) & =\lim _{x \rightarrow \infty} \lambda b(b x)+\bar{\delta}\left[\theta b x-e^{\theta x} \lambda b x\right] \frac{b-\theta e^{\theta x} \frac{b x}{e^{\theta x}}}{e^{\theta x}} \\
& =\lim _{x \rightarrow \infty} \lambda b^{2} x+\bar{\delta}\left[-e^{\theta x} \lambda b x\right]\left[-\frac{\theta b x}{e^{\theta x}}\right]=\lim _{x \rightarrow \infty} \bar{\delta} \lambda \theta b^{2} x^{2}=\infty .
\end{aligned}
$$

Finally, if $b=0$, then $D(x)=c$ and $\Delta(x)=\lambda c>0$. Hence, we get

$$
\lim _{x \rightarrow \infty} k(x)=\lim _{x \rightarrow \infty} \bar{\delta}\left[\theta c-\left(e^{\theta x}-1\right) \lambda c\right] \frac{-\theta e^{\theta x} c}{\left(e^{\theta x}-1\right)^{2}}=\lim _{x \rightarrow \infty} \bar{\delta} \frac{\lambda \theta e^{\theta x} c^{2}}{e^{\theta x}-1}=\bar{\delta} \lambda \theta c^{2}>0 .
$$

From the above results we conclude that if $x$ runs from 0 to $\infty$, then $k(x)$ grows from $-\infty$ to $\infty$ (if $b>0$ ) or to a positive number (if $b=0$ ). We now prove that $k(x)=0$ occurs at most once for $x>0$, by showing that the derivative of $k(x)$ is positive if $k(x)=0$. Using (51), straightforward computations yield

$$
\begin{aligned}
k^{\prime}(x) & =\lambda b^{2}+\bar{\delta}\left[\theta b-\theta e^{\theta x} \Delta(x)-\left(e^{\theta x}-1\right) \lambda b\right] \kappa^{\prime}(x)+\bar{\delta}\left[\theta D(x)-\left(e^{\theta x}-1\right) \Delta(x)\right] \kappa^{\prime \prime}(x) \\
& =\lambda b^{2}+\bar{\delta}\left[\theta b-\theta e^{\theta x} \Delta(x)-\left(e^{\theta x}-1\right) \lambda b\right] \kappa^{\prime}(x)-\frac{\lambda b D(x)}{\kappa^{\prime}(x)} \kappa^{\prime \prime}(x),
\end{aligned}
$$

where the second equality follows from (50). The bracketed expression is negative, because $\left(e^{\theta x}-1\right) \lambda b \geq 0$ and, since $b-\Delta(x)=-\lambda D(x)$,

$$
\theta b-\theta e^{\theta x} \Delta(x)=\theta\left[b-\left(e^{\theta x}-1\right) \Delta(x)-\Delta(x)\right]=\theta\left[-\left(e^{\theta x}-1\right) \Delta(x)-\lambda D(x)\right]<0 .
$$

Since $\kappa^{\prime}(x)<0$ and $\kappa^{\prime \prime}(x)>0$, by Lemma A.1, we may conclude that $k(x)=0$ implies $k^{\prime}(x)>$ 0 , as desired. Hence the proof is complete.

We conclude from Lemma A.2 and Lemma A.3 that $\mathcal{L}^{\prime}(\bar{v})=\mathcal{R}^{\prime}(\bar{v})=0$, where $\bar{v}$ is the unique point where $\mathcal{L}^{\prime}(x)$ and $\mathcal{R}^{\prime}(x)$ vanish. The above proof also reveals that $\mathcal{L}^{\prime}(x)$ and $\mathcal{R}^{\prime}(x)$ change sign at $x=\bar{v}$. Hence, without further proof we may state the next lemma.

Lemma A.4. $\mathcal{L}(x)$ and $\mathcal{R}(x)$ are decreasing if $x<\bar{v}$ and increasing if $x>\bar{v}$.

In the next lemma, we consider the limits of $\mathcal{L}(x)$ and $\mathcal{R}(x)$ when $x$ approaches zero or infinity.

Lemma A.5. ${ }^{3}$ One has

$$
\lim _{x \rightarrow \infty} \mathcal{L}(x)=\infty
$$

Moreover,

$$
\begin{gathered}
\beta>0 \Rightarrow \lim _{x \downarrow 0} \mathcal{L}(x)=\lim _{x \downarrow 0} \mathcal{R}(x)=\lim _{x \rightarrow \infty} \mathcal{R}(x)=\infty, \\
\beta<0 \Rightarrow \lim _{x \downarrow 0} \mathcal{L}(x)=\lim _{x \downarrow 0} \mathcal{R}(x)=\lim _{x \rightarrow \infty} \mathcal{R}(x)=0 .
\end{gathered}
$$

\footnotetext{
${ }^{3}$ It may be mentioned that if $\beta=0$ then the limits are the same as the case when $\beta>0$.
} 
Proof. The proof uses the following facts:

- $\theta>0, \lambda \geq 0$ and $\delta>0$;

- $\bar{\beta}:=\frac{\beta}{\beta+\delta}$

- $\bar{\beta}+\bar{\delta}=1, \bar{\delta}>0$

- $q=\theta \bar{\delta}-\lambda \bar{\beta}$

- $\beta, \bar{\beta}$ and $r$ have the same sign;

- $D(0)=c>0$ and $\Delta(0)=b \lambda c>0$;

Using these properties, the proof becomes more or less straightforward. Using only the sum and product rules for taking limits we may write:

$$
\begin{aligned}
& \lim _{x \downarrow 0} \mathcal{L}(x)=\lim _{x \downarrow 0}\left(\frac{D(0)}{\theta x}\right)^{-\bar{\delta}}\left(\Delta(0)+\frac{\theta \delta}{\beta} \frac{D(0)}{\theta x}\right)=\lim _{x \downarrow 0} \frac{\theta \delta}{\beta}\left(\frac{D(0)}{\theta x}\right)^{\bar{\beta}}, \\
& \lim _{x \rightarrow \infty} \mathcal{L}(x)=\lim _{x \rightarrow \infty}\left(\frac{D(x)}{e^{\theta x}}\right)^{-\bar{\delta}}\left(\Delta(x)+\frac{\theta \delta}{\beta} \frac{D(x)}{e^{\theta x}}\right)=\lim _{x \rightarrow \infty}\left(\frac{e^{\theta x}}{D(x)}\right)^{\bar{\delta}} \Delta(x), \\
& \lim _{x \downarrow 0} \mathcal{R}(x)=\lim _{x \downarrow 0}\left(\frac{D(0)}{\theta x}\right)^{-\bar{\delta}}\left(b+\frac{\theta \delta}{\beta} \frac{D(0)}{\theta x}\right)=\lim _{x \downarrow 0} \frac{\theta \delta}{\beta}\left(\frac{D(0)}{\theta x}\right)^{\bar{\beta}}=\lim _{x \downarrow 0} \mathcal{L}(x), \\
& \lim _{x \rightarrow \infty} \mathcal{R}(x)=\lim _{x \rightarrow \infty}\left(\frac{D(x)}{e^{\theta x}}\right)^{-\bar{\delta}} e^{-q x}\left(b+\frac{\theta \delta}{\beta} D(x)\right)=\lim _{x \rightarrow \infty} \frac{\theta \delta}{\beta} D(x)^{\bar{\beta}} e^{\lambda \bar{\beta} x} .
\end{aligned}
$$

From the above relations one easily deduces the results stated in the lemma. It may be pointed out that for the last limit one needs to use that if $b=0$ then $\lambda>0$, and if $\lambda=0$ then $b>0$, due to (4). This completes the proof.

Lemma A.6. One has $\mathcal{L}(\bar{v})<\mathcal{R}(\bar{v})$. Moreover, $\mathcal{L}(v)=\mathcal{R}(v)$ for some unique number $v>\bar{v}$.

Proof. We have $\mathcal{L}^{\prime}(x)=e^{q x} \mathcal{R}^{\prime}(x)$ and $q>0$. Since $e^{q x}>1$, it follows that if $x<\bar{v}$, then $\mathcal{L}(x)$ decreases faster than $\mathcal{R}(x)$ and if $x>\bar{v}$, then $\mathcal{L}(x)$ increases faster than $\mathcal{R}(x)$. We first deal with the case where $x<\bar{v}$. In that case, we must distinguish the cases $\beta>0$ and $\beta<0$.

We start with $\beta<0$. From Lemma A.5 we know that the limiting value of $\mathcal{L}(x)$ and $\mathcal{R}(x)$ is zero when $x$ approaches zero. Since $\mathcal{L}(x)$ decreases faster than $\mathcal{R}(x)$ if $x \in(0, \bar{v})$, we conclude that $\mathcal{L}(x)<\mathcal{R}(x)$, for all $x<\bar{v}$.

If $\beta>0$, then $\mathcal{L}(x)$ and $\mathcal{R}(x)$ become unbounded when $x$ approaches zero, as we know from Lemma A.5. In order to show that $\mathcal{L}(x)<\mathcal{R}(x)$, for $x<\bar{v}$, it suffices to prove that this holds when $x$ approaches zero. We therefore consider the sign of $\mathcal{R}(x)-\mathcal{L}(x)=\kappa(x)^{-\bar{\delta}}[R(x)-L(x)]$ when $x$ approaches zero. Since $\kappa(x)$ is positive by Lemma A.1, this sign is the same as the sign of

$$
\begin{aligned}
\varepsilon(x) & :=b e^{-q x}+\frac{\theta \delta}{\beta} \kappa(x) e^{r x}-b-\lambda D(x)-\frac{\theta \delta}{\beta} \kappa(x) \\
& =\left(e^{-q x}-1\right) b-\lambda D(x)+\frac{\theta \delta}{\beta} \frac{e^{r x}-1}{e^{\theta x}-1} D(x) .
\end{aligned}
$$


From this, we derive that

$$
\lim _{x \downarrow 0} \varepsilon(x)=\lim _{x \downarrow 0}\left[-\lambda D(x)+\frac{\theta \delta}{\beta} \frac{r}{\theta} D(x)\right]=\left(-\lambda+\frac{\delta r}{\beta}\right) D(0)=q D(0)>0 .
$$

We conclude that if $x<\bar{v}$, then $\mathcal{L}(x)<\mathcal{R}(x)$ holds also when $\beta>0$. This implies, in particular, that $\mathcal{L}(\bar{v})<\mathcal{R}(\bar{v})$.

Finally, we deal with $x>\bar{v}$. Since $\mathcal{L}(x)$ increases exponentially faster than $\mathcal{R}(x)$, for $x \in(\bar{v}, \infty)$, and $\mathcal{L}(x)$ goes to infinity if $x$ grows to infinity, there must exist a number $v>\bar{v}$ such that $\mathcal{L}(v)=$ $\mathcal{R}(v)$. From this point on, $\mathcal{L}(x)$ still grows faster than $\mathcal{R}(x)$, which gives the uniqueness of $v$. This completes the proof of the lemma.

We conclude this section by pointing out another consequence of Lemma A.5, namely,

$$
\beta \mathcal{R}(x)>0, \quad \forall x>0
$$

This is obvious if $\beta>0$, because then the definition of $\mathcal{R}$ implies $\mathcal{R}(x)>0$, for all $x>0$. If $\beta<$ 0 , then Lemma A.5 gives that $\mathcal{R}(x)$ converges to zero both when $x$ approaches zero and when $x$ grows to infinity. Due to Lemma A.4, this implies that $\mathcal{R}(x)<0$, for all $x>0$.

\section{Appendix B. Objective Value in the Unhealthy Case}

Taking the sum of all stationarity conditions (5) for $t_{k}(k \geq 2)$, we get

$$
\sum_{k=2}^{\infty} \delta D\left(u_{k}\right) e^{\lambda h_{k}-\delta t_{k}}=\sum_{k=2}^{\infty}\left(S_{0} e^{\beta t_{k}-\theta h_{k-1}}-S_{0} e^{\beta t_{-} k-\theta h_{k}}\right) .
$$

This yields a linear relation between $I(u, \tau)$ and $A(u, \tau)$ at a stationary point as follows:

$$
\begin{aligned}
\delta\left(I(u, \tau)-D\left(u_{1}\right) e^{\lambda u_{1}}\right) & =\sum_{k=2}^{\infty} S_{0} e^{\beta t_{k}-\theta h_{k-1}}-\sum_{k=3}^{\infty} S_{0} e^{\beta t_{k-1}-\theta h_{k-1}} \\
& =\sum_{k=2}^{\infty} S_{0} e^{\beta t_{k}-\theta h_{k-1}}+S_{0} e^{\beta t_{1}-\theta h_{1}}-\sum_{k=2}^{\infty} S_{0} e^{\beta t_{k-1}-\theta h_{k-1}} \\
& =S_{0} e^{\beta t_{1}-\theta h_{1}}+\sum_{k=2}^{\infty} S_{0}\left[e^{\beta t_{k}}-e^{\beta t_{k-1}}\right] e^{-\theta h_{k-1}} \\
& =S_{0} e^{-\theta u_{1}}+\beta\left(A(u, \tau)-S_{0}\left[e^{\beta t_{1}}-e^{\beta t_{0}}\right] e^{-\theta h_{0}}\right) \\
& =S_{0} e^{-\theta u_{1}}+\beta A(u, \tau),
\end{aligned}
$$

where we used $t_{0}=t_{1}=h_{0}=0$. The stationarity condition (13) for $u_{2}$ yields

$$
b e^{\lambda h_{2}-\delta t_{2}}+\sum_{\ell=2}^{\infty} \lambda D\left(u_{\ell}\right) e^{\lambda h_{\ell}-\delta t_{\ell}}=\sum_{\ell=3}^{\infty} \frac{\theta S_{0}}{\beta}\left(e^{\beta t_{\ell}}-e^{\beta t_{\ell-1}}\right) e^{-\theta h_{\ell-1}} .
$$


This implies

$$
b e^{\lambda h_{2}-\delta t_{2}}+\lambda\left[I(u, \tau)-D\left(u_{1}\right) e^{\lambda h_{1}-\delta t_{1}}\right]=\theta\left[A(u, \tau)-\frac{S_{0}}{\beta}\left(e^{\beta t_{2}}-e^{\beta t_{1}}\right) e^{-\theta h_{1}}\right],
$$

where we used once more that $\left(e^{\beta t_{1}}-e^{\beta t_{0}}\right) e^{-\theta h_{0}}=0$. Since $h_{1}=u_{1}$ and $t_{1}=0$, this gives a second linear relation between $I(u, \tau)$ and $A(u, \tau)$, namely,

$$
b e^{\lambda h_{2}-\delta t_{2}}+\sum_{\ell=2}^{\infty} \lambda D\left(u_{\ell}\right) e^{\lambda h_{\ell}-\delta t_{\ell}}=\sum_{\ell=3}^{\infty} \frac{\theta S_{0}}{\beta}\left(e^{\beta t_{\ell}}-e^{\beta t_{\ell-1}}\right) e^{-\theta h_{\ell-1}}
$$

Since $\beta t_{2}-\theta u_{1}=\beta t_{2}-\theta\left(h_{2}-u_{2}\right)$, we may reduce this equality as follows:

$$
\begin{aligned}
-\lambda I(u, \tau)+\theta A(u, \tau) & =b e^{\lambda h_{2}-\delta t_{2}}+\frac{\theta S_{0}}{\beta} e^{\beta t_{2}-\theta h_{2}} e^{\theta u_{2}}-\lambda D\left(u_{1}\right) e^{\lambda u_{1}}-\frac{\theta S_{0}}{\beta} e^{-\theta u_{1}} \\
& =b e^{\lambda h_{2}-\delta t_{2}}+\frac{\theta \delta}{\beta} \kappa\left(u_{2}\right) e^{\lambda h_{2}-\delta t_{2}} e^{\theta u_{2}}-\lambda D\left(u_{1}\right) e^{\lambda u_{1}}-\frac{\theta S_{0}}{\beta} e^{-\theta u_{1}} \\
& =\left(b+\frac{\theta \delta}{\beta} \kappa\left(u_{2}\right) e^{\theta u_{2}}\right) e^{\lambda h_{2}-\delta t_{2}}-\lambda D\left(u_{1}\right) e^{\lambda u_{1}}-\frac{\theta S_{0}}{\beta} e^{-\theta u_{1}} \\
& =e^{q u_{2}} R\left(u_{2}\right) e^{\lambda h_{2}-\delta t_{2}}-\lambda D\left(u_{1}\right) e^{\lambda u_{1}}-\frac{\theta S_{0}}{\beta} e^{-\theta u_{1}}
\end{aligned}
$$

where the second equality is due to (12), i.e., stationarity with respect to $t_{2}$. Using stationarity with respect to $t_{2}$ once more, we get, from (17),

$$
e^{-\delta t_{2}}=\left(\frac{S_{0}}{\delta}\right)^{\bar{\delta}} \kappa\left(u_{2}\right)^{-\bar{\delta}} e^{-(q+\lambda) h_{2}}
$$

Substitution gives

$$
\begin{aligned}
-\lambda I(u, \tau)+\theta A(u, \tau) & =e^{q u_{2}} R\left(u_{2}\right)\left(\frac{S_{0}}{\delta}\right)^{\bar{\delta}} \kappa\left(u_{2}\right)^{-\bar{\delta}} e^{-q h_{2}}-\lambda D\left(u_{1}\right) e^{\lambda u_{1}}-\frac{\theta S_{0}}{\beta} e^{-\theta u_{1}} \\
& =\left(\frac{S_{0}}{\delta}\right)^{\bar{\delta}} \kappa\left(u_{2}\right)^{-\bar{\delta}} R\left(u_{2}\right) e^{-q u_{1}}-\lambda D\left(u_{1}\right) e^{\lambda u_{1}}-\frac{\theta S_{0}}{\beta} e^{-\theta u_{1}} \\
& =\left(\frac{S_{0}}{\delta}\right)^{\bar{\delta}} \mathcal{R}\left(u_{2}\right) e^{-q u_{1}}-\lambda D\left(u_{1}\right) e^{\lambda u_{1}}-\frac{\theta S_{0}}{\beta} e^{-\theta u_{1}}
\end{aligned}
$$

Thus, we have obtained two linear relations between $I(u, \tau)$ and $A(u, \tau)$, namely,

$$
\begin{aligned}
\delta I(u, \tau)-\beta A(u, \tau) & =\delta D\left(u_{1}\right) e^{\lambda u_{1}}+S_{0} e^{-\theta u_{1}} \\
-\lambda I(u, \tau)+\theta A(u, \tau) & =\left(\frac{S_{0}}{\delta}\right)^{\delta} \mathcal{R}\left(u_{2}\right) e^{-q u_{1}}-\lambda D\left(u_{1}\right) e^{\lambda u_{1}}-\theta \frac{S_{0}}{\beta} e^{-\theta u_{1}}
\end{aligned}
$$


Just as in Section 5 for the healthy case, this system has a unique solution. By solving the system for $I(u, \tau)$ and $A(u, \tau)$, we get, in the same way as in Section 5, the following expressions:

$$
\begin{aligned}
& (\theta \delta-\beta \lambda) A(u, \tau)=\delta\left(\frac{S_{0}}{\delta}\right)^{\bar{\delta}} \mathcal{R}\left(u_{2}\right) e^{-q u_{1}}-\frac{(\theta \delta-\lambda \beta) S_{0}}{\beta} e^{-\theta u_{1}} \\
& (\theta \delta-\beta \lambda) I(u, \tau)=(\theta \delta-\beta \lambda) D\left(u_{1}\right) e^{\lambda u_{1}}+\beta\left(\frac{S_{0}}{\delta}\right)^{\bar{\delta}} \mathcal{R}\left(u_{2}\right) e^{-q u_{1}}
\end{aligned}
$$

By adding these equations, we obtain the value of $f(u, \tau)$ at a stationary point:

$$
f(u, \tau)=\frac{e^{-q u_{1}}}{q}\left(\frac{S_{0}}{\delta}\right)^{\bar{\delta}} \mathcal{R}\left(u_{2}\right)+D\left(u_{1}\right) e^{\lambda u_{1}}-\frac{S_{0}}{\beta} e^{-\theta u_{1}} .
$$

\section{Appendix C. Data of 22 Dikes in The Netherlands}

Table 2 contains the raw data of the 22 dikes in The Netherlands for which the data are available.

Table 3 contains the values of the parameters that play a role in the theoretical analysis.

It was shown in Section 8 that the 12 dikes numbered 10, 11, 16, 22, 23, 24, 40, 42, 43, 49, 51, and 52 were healthy and the other 10 dikes were unhealthy. 
Table 2. Raw data for the 22 dikes.

\begin{tabular}{rrrrrrrrrr}
\hline No. & $c$ & $b$ & $\lambda$ & $\sigma$ & $\eta$ & $\gamma$ & $\zeta$ & $P_{0}$ & $V_{0}$ \\
\hline 10 & 16.6939 & 0.6258 & 0.0014 & 0.033027 & 0.320 & 0.02 & 0.003774 & $1 / 2270$ & 1564.9 \\
11 & 42.6200 & 1.7068 & 0.0000 & 0.032000 & 0.320 & 0.02 & 0.003469 & $1 / 855$ & 1700.1 \\
15 & 125.6422 & 1.1268 & 0.0098 & 0.050200 & 0.760 & 0.02 & 0.003764 & $1 / 729$ & 11810.4 \\
16 & 324.6287 & 2.1304 & 0.0100 & 0.057400 & 0.760 & 0.02 & 0.002032 & $1 / 906$ & 22656.5 \\
22 & 154.4388 & 0.9325 & 0.0066 & 0.070000 & 0.620 & 0.02 & 0.002893 & $1 / 1802$ & 9641.1 \\
23 & 26.4653 & 0.5250 & 0.0034 & 0.053400 & 0.800 & 0.02 & 0.002031 & $1 / 729$ & 61.6 \\
24 & 71.6923 & 1.0750 & 0.0059 & 0.043900 & 1.060 & 0.02 & 0.003733 & $1 / 531$ & 2706.4 \\
35 & 49.7384 & 0.6888 & 0.0088 & 0.036000 & 1.060 & 0.02 & 0.004105 & $1 / 509$ & 4534.7 \\
38 & 24.3404 & 0.7000 & 0.0040 & 0.025321 & 0.412 & 0.02 & 0.004153 & $1 / 585$ & 3062.6 \\
40 & 5.8601 & 0.1000 & 0.0026 & 0.025321 & 0.422 & 0.02 & 0.003905 & $1 / 855$ & 43.5 \\
41 & 58.8110 & 0.9250 & 0.0033 & 0.025321 & 0.422 & 0.02 & 0.002794 & $1 / 585$ & 10013.1 \\
42 & 21.8254 & 0.4625 & 0.0019 & 0.026194 & 0.442 & 0.02 & 0.001241 & $1 / 585$ & 1090.8 \\
43 & 340.5081 & 4.2975 & 0.0043 & 0.025321 & 0.448 & 0.02 & 0.002043 & $1 / 585$ & 19767.6 \\
44 & 24.0977 & 0.7300 & 0.0054 & 0.031651 & 0.316 & 0.02 & 0.003485 & $1 / 3050$ & 37596.3 \\
45 & 3.4375 & 0.1375 & 0.0069 & 0.033027 & 0.320 & 0.02 & 0.002397 & $1 / 6120$ & 10421.2 \\
47 & 8.7813 & 0.3513 & 0.0026 & 0.029000 & 0.358 & 0.02 & 0.003257 & $1 / 585$ & 1369.0 \\
48 & 35.6250 & 1.4250 & 0.0063 & 0.023019 & 0.496 & 0.02 & 0.003076 & $1 / 585$ & 7046.4 \\
49 & 20.0000 & 0.8000 & 0.0046 & 0.034529 & 0.304 & 0.02 & 0.003744 & $1 / 585$ & 823.3 \\
50 & 8.1250 & 0.3250 & 0.0000 & 0.033027 & 0.320 & 0.02 & 0.004033 & $1 / 585$ & 2118.5 \\
51 & 15.0000 & 0.6000 & 0.0071 & 0.036173 & 0.294 & 0.02 & 0.004315 & $1 / 585$ & 570.4 \\
52 & 49.2200 & 1.6075 & 0.0047 & 0.036173 & 0.304 & 0.02 & 0.001716 & $1 / 585$ & 4025.6 \\
53 & 69.4565 & 1.1625 & 0.0028 & 0.031651 & 0.336 & 0.02 & 0.002700 & $1 / 585$ & 9819.5 \\
\hline
\end{tabular}


Table 3. Data used in the analysis, for the 22 dikes

\begin{tabular}{rrrrrrrrr}
\hline no. & $c$ & $b$ & $\lambda$ & $\beta$ & $\theta$ & $\delta$ & $q$ & $v$ \\
\hline 10 & 16.6939 & 0.6258 & 0.0014 & -0.0094 & 0.0293 & 0.04 & 0.0387 & 56.9610 \\
11 & 42.6200 & 1.7068 & 0.0000 & -0.0098 & 0.0285 & 0.04 & 0.0377 & 62.4217 \\
15 & 125.6422 & 1.1268 & 0.0098 & 0.0182 & 0.0464 & 0.04 & 0.0289 & 53.2922 \\
16 & 324.6287 & 2.1304 & 0.0100 & 0.0236 & 0.0554 & 0.04 & 0.0311 & 52.5944 \\
22 & 154.4388 & 0.9325 & 0.0066 & 0.0234 & 0.0671 & 0.04 & 0.0399 & 53.7019 \\
23 & 26.4653 & 0.5250 & 0.0034 & 0.0227 & 0.0514 & 0.04 & 0.0315 & 55.2417 \\
24 & 71.6923 & 1.0750 & 0.0059 & 0.0265 & 0.0402 & 0.04 & 0.0218 & 61.8285 \\
35 & 49.7338 & 0.6888 & 0.0088 & 0.0182 & 0.0319 & 0.04 & 0.0192 & 59.6453 \\
38 & 24.3404 & 0.7000 & 0.0040 & -0.0096 & 0.0212 & 0.04 & 0.0291 & 62.0429 \\
40 & 5.8601 & 0.1000 & 0.0026 & -0.0093 & 0.0214 & 0.04 & 0.0287 & 78.8044 \\
41 & 55.8110 & 0.9250 & 0.0033 & -0.0093 & 0.0226 & 0.04 & 0.0304 & 74.6588 \\
42 & 21.8254 & 0.4625 & 0.0019 & -0.0084 & 0.0250 & 0.04 & 0.0321 & 72.2395 \\
43 & 340.5081 & 4.2975 & 0.0043 & -0.0087 & 0.0233 & 0.04 & 0.0309 & 73.4901 \\
44 & 24.0977 & 0.7300 & 0.0054 & -0.0100 & 0.0282 & 0.04 & 0.0394 & 49.5379 \\
45 & 3.4375 & 0.1375 & 0.0069 & -0.0094 & 0.0306 & 0.04 & 0.0422 & 41.4883 \\
47 & 8.7813 & 0.3513 & 0.0026 & -0.0096 & 0.0257 & 0.04 & 0.0347 & 55.5986 \\
48 & 35.6250 & 1.4250 & 0.0063 & -0.0086 & 0.0199 & 0.04 & 0.0271 & 50.8446 \\
49 & 20.0000 & 0.8000 & 0.0046 & -0.0095 & 0.0308 & 0.04 & 0.0418 & 54.6507 \\
50 & 8.1250 & 0.3250 & 0.0000 & -0.0094 & 0.0290 & 0.04 & 0.0379 & 61.9738 \\
51 & 15.0000 & 0.6000 & 0.0071 & -0.0094 & 0.0319 & 0.04 & 0.0438 & 40.4924 \\
52 & 49.2200 & 1.6075 & 0.0047 & -0.0090 & 0.0345 & 0.04 & 0.0458 & 45.7435 \\
53 & 69.4565 & 1.1625 & 0.0028 & -0.0094 & 0.0290 & 0.04 & 0.0387 & 66.1279 \\
\hline
\end{tabular}

